Research Article

\title{
Free Vibration Analysis of Rotating Pretwisted Functionally Graded Sandwich Blades
}

\author{
L. T. Liu $\mathbb{D},{ }^{1}$ Y. X. Hao $\left(\mathbb{D},{ }^{1}\right.$ W. Zhang $\left(\mathbb{D},{ }^{2}\right.$ and J. Chen $\mathbb{D}^{3}$ \\ ${ }^{1}$ College of Mechanical Engineering, Beijing Information Science and Technology University, Beijing 100192, China \\ ${ }^{2}$ College of Mechanical Engineering, Beijing University of Technology, Beijing 100124, China \\ ${ }^{3}$ Architecture and Civil Engineering Research Center, Shenzhen Research Institute, City University of Hong Kong, Shenzhen, China
}

Correspondence should be addressed to Y.X. Hao; bimhao@163.com

Received 6 October 2017; Revised 12 June 2018; Accepted 12 July 2018; Published 16 September 2018

Academic Editor: Ratneshwar Jha

Copyright @ 2018 L. T. Liu et al. This is an open access article distributed under the Creative Commons Attribution License, which permits unrestricted use, distribution, and reproduction in any medium, provided the original work is properly cited.

A new structural dynamic model for the free vibration characteristic analysis of rotating pretwisted functionally graded (FG) sandwich blades is developed. The sandwich blade is made up of two functionally graded skins and a homogeneous material core. The thick shell theory is applied to derive the basic equations of motion of the rotating FG sandwich blade by considering the effects of centrifugal and Coriolis forces. The mode shapes are expanded in terms of two-dimensional algebraic polynomials in the Rayleigh-Ritz method, and the static and dynamic natural frequencies of the blade are obtained. The convergence analysis is studied, and the accuracy of the proposed model is verified by comparing with the literature results and ANSYS data. The effects of frequency parameters such as the twist angle, the thickness ratio, the aspect ratio, the layer thickness ratio, the scalar parameter of volume fraction, the stagger angle, and the rotation velocity on the vibration characteristics for pretwist FG sandwich blade are investigated in detail. In addition, the phenomena of frequency locus veering and mode shape exchanging occur in the static and dynamic states. Frequency locus veering is essentially caused by the coupling between different modes.

\section{Introduction}

The aero gas turbine engine is the key technology of the aircraft; blade is one of the most important parts of the system. Vibration, especially resonance of the blade, will produce larger stress, which will lead to fatigue failure. Thus, the dynamic behavior of rotating blades has been studied by numerous researchers.

Some simplified blade models such as beams, plates, and shells are used to investigate the vibration characteristics of the blades in the published literatures. Leissa and Jacob [1] investigated the free vibration of pretwisted, cantilevered beams and plates by using the Ritz method. The work was the first three-dimensional study of the problem. Yoo et al. [2] derived the equations of motion with a concentrated mass by using a modeling method with hybrid deformation variables and investigated the effects of the nondimensional parameters on the vibration characteristics of the pretwisted rotating blade through numerical analysis. Chandiramani et al. [3] simplified the pretwisted rotating blade as a laminated composite, hollow uniform box-beam model, which considers the centrifugal and Coriolis effects, transverse shear flexibility, and restrained warping, and studied the free and forced vibration by using HSDT and Galerkin method. Carrera et al. [4] used the Carrera unified formulation and FEM to study the free vibration analysis of rotating blades; the Coriolis and centrifugal force fields were included in their work.

The beam models are quite suitable for blades with large aspect ratio and low width thickness ratio. However, flexible rotating structures such as blades with low aspect ratios are also widely used in actual engineering applications. Therefore, more and more literatures were found to study the vibration of blade with plate and shell models.

Qatu and Leissa [5] were the first to study the effect of plate parameters such as twist angle, stacking sequence, and lamination angle on the natural frequency and mode shapes of laminated composite pretwisted cantilever plates by using the shallow shell theory and Ritz method. Nabi and Ganesan [6] analyzed the vibration characteristics of metal matrix 
composite pretwisted blades by using beam and plate theories, respectively, and summed up the quantitative comparison of natural frequency. Yoo and Chung [7] developed a linear dynamic modeling method for plates by using two in-plane stretch variables and analyzed and studied the transient characteristics of rotating plates. Hu et al. [8] proposed a numerical procedure for the free vibration analysis of pretwisted thin plates based on the thin shell theory and studied the vibration characteristics considering different twist rates and aspect ratios. Hashemi et al. [9] used the Mindlin plate theory and Kane dynamic method to develop a finite element formulation for vibration analysis of rotating thick plates. The coupling between in-plane and out-of-plane deformations and Coriolis effect was considered in the study. Sinha and Turner [10] derived the governing partial differential equation of motion for the rotating pretwisted plate by using the thin shell theory and studied the free vibration of a turbomachinery cantilevered airfoil blade with the Rayleigh-Ritz technique by considering the centrifugal force filed as a quasi-static load. Sun et al. [11] presented a dynamic model based on CLPT, investigated the vibration behavior of a rotating blade by using Hamilton's principle, and studied the point and distribution forced response using a proportional damping model.

Rao and Gupta [12] studied the vibration of natural frequencies with various parameters of a rotating pretwisted blade with a small aspect ratio by using the classical bending theory of thin shells. Sun et al. [13] developed a novel dynamic model for pretwisted rotating compressor blades by using the general shell theory and studied the eigenfrequencies and damping properties of the pretwisted rotating blade. Sinha and Zylka [14] derived the governing partial differential equation of motion based on the thin shell theory and formulated the free vibration of a turbomachinery cantilevered airfoil by considering it as an anisotropic shell in a centrifugal force field. Kielb et al. [15] gave the complete theoretical and experimental results of a joint research study on the vibration characteristics of pretwisted cantilever plates.

Claassen and Thorne [16] were the first to find out and discuss the curve veering in the plate vibration based on CLPT. Afolabi and Mehmed [17] studied the curve veering and flutter of rotating blades based on the theory of algebraic curves and catastrophe theory. They found that the frequency loci of undamped rotating blades do not cross, but must either repel each other or attract each other. Yoo and Pierre $[18,19]$ studied the vibration characteristics of rotating cantilever rectangular plates and composite plates, respectively, and found out and discussed the natural frequency locus veering, crossing, and associated mode shape variations in detail.

Most of the early studies were focused on homogeneous materials, and in recent years, some researchers have extended the range to composite materials because of their excellent properties. The structure was developed from single layer to multilayer and composite layer, and more and more literature has been published.

Oh et al. [20] studied the vibration of turbomachinery rotating blades made up of functionally graded materials and investigated the influence of parameters on the frequency of rotating blades. A refined dynamic theory involving the coupling between flapping, lagging, and transverse shear was used in their work. Ramesh and Rao [21] studied the natural frequencies of a pretwisted rotating FG cantilever beam by using Lagrange's equation and the Rayleigh-Ritz method. The influences of different parameters and coupling between chordwise and flapwise bending modes on the natural frequency were investigated. Oh and Yoo [22] presented a new structural dynamic model to study the vibration characteristics of rotating blades. The blade was modeled as a functionally graded material pretwisted beam by using the Rayleigh-Ritz and Kane method. Mantari and Granados [23] studied the free vibration of FG plates by using a new novel FSDT with 4 unknowns. Li and Zhang [24] developed a dynamic model for FG plates undergoing large overall motions, studied the free vibration of rotating cantilever FGM rectangular plates, and found frequency locus veering and associated mode shift phenomena. Sun et al. [25] developed a novel dynamic model for multilayer blades by using the quadratic layerwise theory and studied the structural dynamics, particularly the damping properties of the rotating blade. Frequency locus veering with rotation speed was also found. Cao et al. [26] developed a rotating cantilever pretwisted sandwich plate model with a prestagger angle and investigated the vibrational behavior of a turbine blade with thermal barrier coating layers by using FSDT, von Karman plate theory, and Chebyshev-Ritz method.

In the present paper, a new structural dynamic model is developed to study the free vibration characteristics of pretwisted rotating FG sandwich blades. The thick shell theory is applied to derive the basic equations of motion by considering the effects of centrifugal and Coriolis forces. The accuracy of the proposed model is verified by comparing with the literature and ANSYS. The effects of frequency parameters such as the twist angle, the thickness ratio, the aspect ratio, the layer thickness ratio, the scalar parameter of volume fraction, the stagger angle, and the rotation velocity on the vibration characteristics are investigated in detail. Furthermore, frequency locus veering and mode shape exchanging phenomena are found both in both static and dynamic states.

\section{Theoretical Analysis}

2.1. Basic Equations. As shown in Figure 1, the functionally graded sandwich blade is modeled as a cantilever pretwisted thick plate with the twist angle $\theta$ at the free end, which is clamped to the rigid disk with a radius $R$, mounted with a stagger angle $\varphi$. The geometric parameters of the blade are the span (length) dimension $L$, the chord (width) dimension $b$, the total thickness $h$, and the angular rotating velocity about the rigid disk axis $\Omega$.

In this paper, three coordinate systems are established for dynamic modeling. One is the $X Y Z$-coordinate system (with unit vectors $\left.\left(\mathbf{i}_{X}, \mathbf{i}_{Y}, \mathbf{i}_{Z}\right)\right)$, where the $x$-axis is along the spanwise direction of the blade, $Y$ is the rotation axis, and the $Z$-axis is perpendicular to the $X Y$-plane following the right-hand rule. The other is the $x y z$-coordinate system (with unit vectors $\left(\mathbf{i}_{x}, \mathbf{i}_{y}, \mathbf{i}_{z}\right)$ ), where the origin lies in the root of the mid-surface of the blade and the $x$-axis is parallel to the $X$-axis; the $y$-axis can be obtained by rotating around the $x$-axis with the stagger angle $\varphi$ starting from the $Y$-axis 


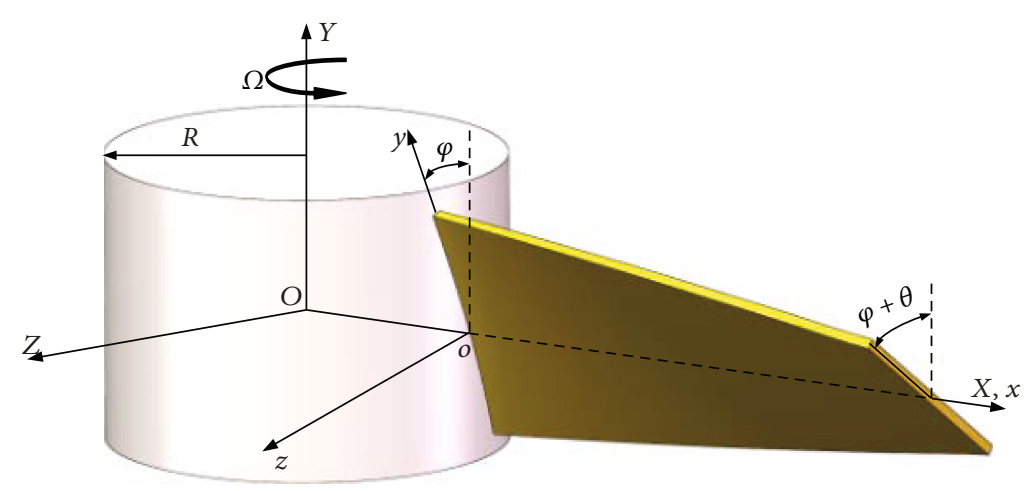

Figure 1: A typical pretwisted rotating blade model.

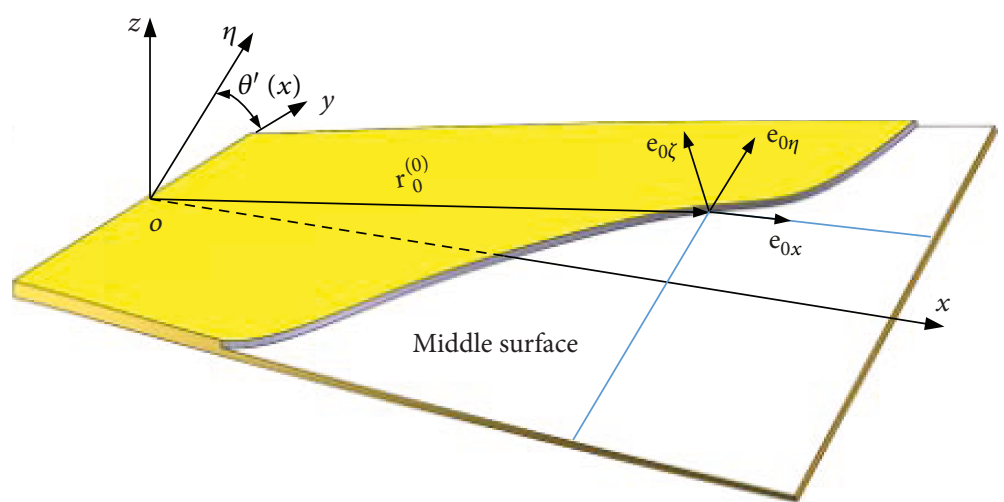

FIGURE 2: The dynamic coordinate system of the pretwisted rotating blade.

direction. Figure 2 shows the last $x \eta$ coordinate, a dynamic surface coordinate where the $\eta$-axis is perpendicular to the $x$-axis and equal to the $y$-axis at $x=0$, rotating at a uniform twist rate $k=\theta / L$. Thus, the rotating angle at $x$ can be expressed as

$$
\theta^{\prime}(x)=k x, \quad x \in[0, L]
$$

The position vector $\mathbf{r}_{0}^{(0)}(x, \eta)$ of a typical point on the mid-surface of the pretwisted blade in the $x \eta$-coordinate can be written as

$$
\mathbf{r}_{0}^{(0)}(x, \eta)=\left[\begin{array}{c}
x \\
\eta \cos \theta^{\prime} \\
\eta \sin \theta^{\prime}
\end{array}\right]^{T}\left(\begin{array}{c}
\mathbf{i}_{x} \\
\mathbf{i}_{y} \\
\mathbf{i}_{z}
\end{array}\right)
$$

Based on the Frenet-Serret formula [28], the corresponding unit base vectors can be expressed as

$$
\left(\begin{array}{c}
\mathbf{e}_{0 x} \\
\mathbf{e}_{0 \eta} \\
\mathbf{e}_{0 \zeta}
\end{array}\right)=\frac{1}{A}\left[\begin{array}{rrr}
1 & -k \eta \sin \theta^{\prime} & k \eta \cos \theta^{\prime} \\
0 & A \cos \theta^{\prime} & A \sin \theta^{\prime} \\
-k \eta & -\sin \theta^{\prime} & \cos \theta^{\prime}
\end{array}\right]\left(\begin{array}{c}
\mathbf{i}_{x} \\
\mathbf{i}_{y} \\
\mathbf{i}_{z}
\end{array}\right),
$$

where $A$ is the Lame parameter of the middle surface in the $x$-direction, given by

$$
A=\sqrt{1+k^{2} \eta^{2}}
$$

Then, an arbitrary point of the blade before deformation can be expressed by a position vector $\mathbf{r}^{(0)}$ as

$$
\mathbf{r}^{(0)}=\mathbf{r}_{0}^{(0)}+\zeta \mathbf{e}_{0 \zeta}=\left[\begin{array}{c}
x-k \eta \frac{\zeta}{A} \\
\eta \cos \theta^{\prime}-\zeta \sin \frac{\theta^{\prime}}{A} \\
\eta \sin \theta^{\prime}+\zeta \cos \frac{\theta^{\prime}}{A}
\end{array}\right]^{T}\left(\begin{array}{c}
\mathbf{i}_{x} \\
\mathbf{i}_{y} \\
\mathbf{i}_{z}
\end{array}\right) .
$$

As illustrated in Figure 3, the core of the blade $\left(h_{1} \leq \zeta \leq h_{2}\right)$ is fully metal (isotropic) and two skins are composed of functionally graded material (FGM) in the thickness direction. The top skin varies from ceramic-rich surface $\left(\zeta=h_{3}\right)$ to metal-rich surface $\left(\zeta=h_{2}\right)$, while the bottom skin varies from ceramic-rich surface $\left(\zeta=h_{0}\right)$ to metal-rich surface $\left(\zeta=h_{1}\right)$. There are no interfaces between the core and skins of the functionally graded sandwich blade, and two skins are symmetrical about the middle 


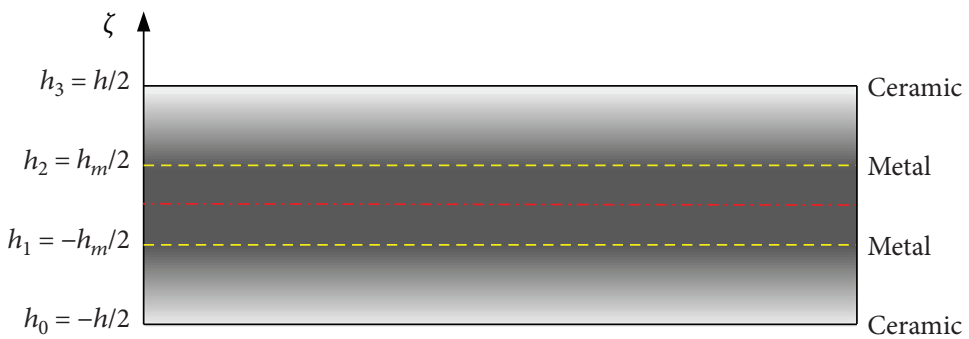

FIgURE 3: The section of the FG sandwich blade.

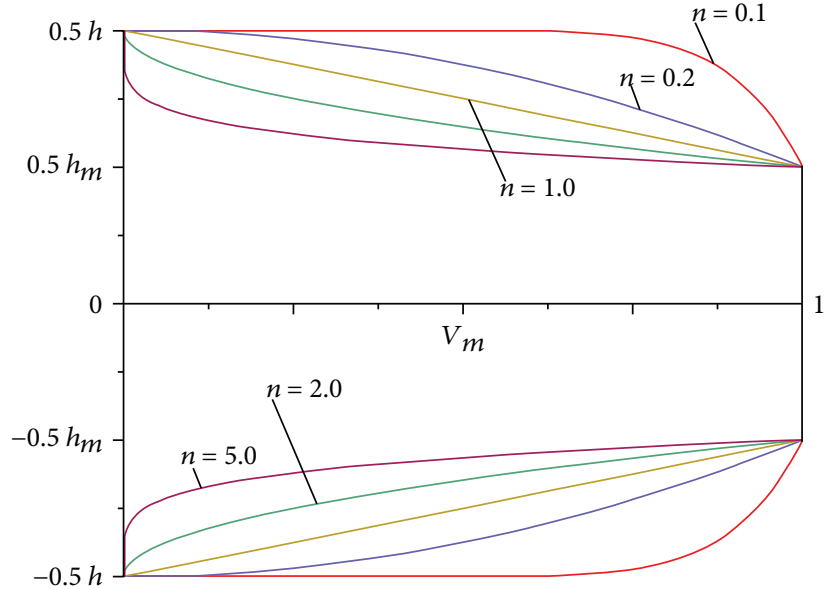

(a) $h_{m}: h=0.5$

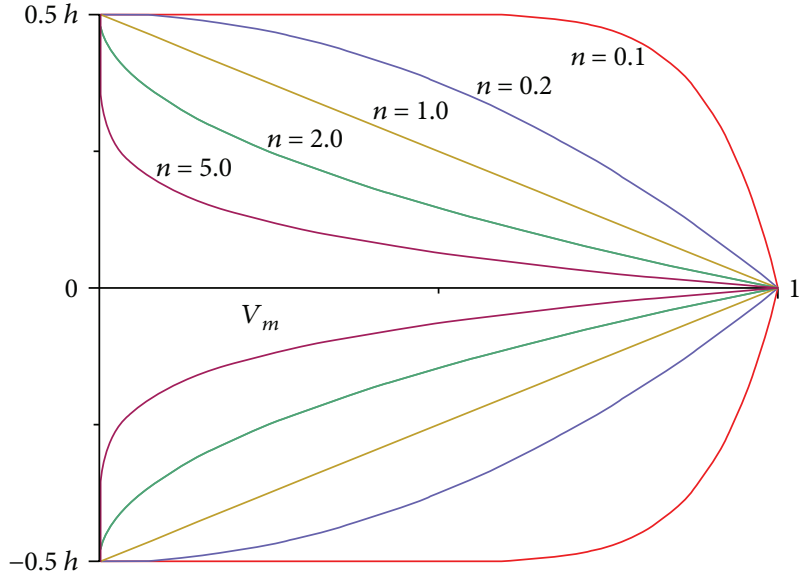

(b) $h_{m}=0$

FIGURE 4: Effect of the scalar parameter of volume fraction in the FG sandwich blade.

surface in this paper. The volume fraction of the metal phase is obtained as [27]

$$
V_{m}(\zeta)=\left\{\begin{array}{cc}
\left(\frac{h+2 \zeta}{h-h_{m}}\right)^{n} & \zeta \in\left[h_{0}, h_{1}\right], \\
1 & \zeta \in\left[h_{1}, h_{2}\right], \\
\left(\frac{h-2 \zeta}{h-h_{m}}\right)^{n} & \zeta \in\left[h_{2}, h_{3}\right] .
\end{array}\right.
$$

As shown in Figure $4, n$ is the scalar parameter that allows users to define the gradation of material properties in the thickness direction. The $n=0$ case corresponds to a fully metal blade. The volume fraction for the ceramic phase is given as

$$
V_{c}(\zeta)=1-V_{m}(\zeta)
$$

In the present work, the homogenization procedure and the law of mixtures are used. The variation of the parameters in the thickness direction is given by

$$
P(\zeta)=P_{\mathrm{m}} V_{m}(\zeta)+P_{\mathrm{c}} V_{c}(\zeta)
$$

where $P(\zeta)$ is the physical parameter, which refers to density $\rho$, elastic modulus $E$, and Poisson's ratio $\mu$. In this paper, $P_{\mathrm{m}}$ and $P_{\mathrm{c}}$ are the physical parameters of metal and ceramic phase.

2.2. The Strain Energy. According to the first-order shear deformation theory, the displacements can be written as

$$
\begin{aligned}
u(x, \eta, \zeta) & =u_{0}(x, \eta)+\zeta \phi_{x}(x, \eta) \\
v(x, \eta, \zeta) & =v_{0}(x, \eta)+\zeta \phi_{\eta}(x, \eta) \\
w(x, \eta, \zeta) & =w_{0}(x, \eta)
\end{aligned}
$$

where $\phi_{x}$ and $\phi_{\eta}$ are mid-surface rotations and $u_{0}, v_{0}$, and $w_{0}$ are mid-surface displacements of the blade along the $x, \eta$, and $\zeta$ directions.

According to the thick shell theory [29] and the improved version of the Novozhilov nonlinear shell theory [30], the strains at any point in the blade can be written as

$$
\begin{aligned}
& \left(\begin{array}{c}
\varepsilon_{x} \\
\varepsilon_{\eta} \\
\gamma_{x \eta}
\end{array}\right)=\left(\begin{array}{c}
\varepsilon_{x}^{(0)} \\
\varepsilon_{\eta}^{(0)} \\
\gamma_{x \eta}^{(0)}
\end{array}\right)+\frac{2}{R_{x \eta}}\left(\begin{array}{c}
0 \\
0 \\
w_{0}
\end{array}\right)+\zeta\left(\begin{array}{c}
\kappa_{x}^{(1)} \\
\kappa_{\eta}^{(1)} \\
\kappa_{x \eta}^{(1)}
\end{array}\right), \\
& \left(\begin{array}{l}
\gamma_{x \zeta} \\
\gamma_{\eta \zeta}
\end{array}\right)=\left(\begin{array}{c}
\gamma_{x \zeta}^{(0)} \\
\gamma_{\eta \zeta}^{(0)}
\end{array}\right)-\frac{1}{R_{x \eta}}\left(\begin{array}{l}
v_{0} \\
u_{0}
\end{array}\right),
\end{aligned}
$$


where

$$
\begin{aligned}
& \left(\begin{array}{c}
\varepsilon_{x}^{(0)} \\
\varepsilon_{\eta}^{(0)} \\
\gamma_{x \eta}^{(0)}
\end{array}\right)=\left(\begin{array}{c}
\frac{1}{A} \frac{\partial u_{0}}{\partial x}+\frac{v_{0}}{A} \frac{\partial A}{\partial \eta}+\frac{1}{2}\left(\frac{1}{A} \frac{\partial w_{0}}{\partial x}\right)^{2} \\
\frac{\partial v_{0}}{\partial \eta}+\frac{1}{2}\left(\frac{\partial w_{0}}{\partial \eta}\right)^{2} \\
\frac{\partial u_{0}}{\partial \eta}+\frac{1}{A} \frac{\partial v_{0}}{\partial x}-\frac{u_{0}}{A} \frac{\partial A}{\partial \eta}+\frac{1}{A} \frac{\partial w_{0}}{\partial x} \frac{\partial w_{0}}{\partial \eta}
\end{array}\right) \\
& \left(\begin{array}{c}
\kappa_{x}^{(1)} \\
\kappa_{\eta}^{(1)} \\
\kappa_{x \eta}^{(1)}
\end{array}\right)=\left(\begin{array}{c}
\frac{1}{A} \frac{\partial \phi_{x}}{\partial x}+\frac{\phi_{\eta}}{A} \frac{\partial A}{\partial \eta} \\
\frac{\partial \phi_{\eta}}{\partial \eta} \\
\frac{\partial \phi_{x}}{\partial \eta}+\frac{1}{A} \frac{\partial \phi_{\eta}}{\partial x}-\frac{\phi_{x}}{A} \frac{\partial A}{\partial \eta}
\end{array}\right) \\
& \left(\begin{array}{c}
\gamma_{x \zeta}^{(0)} \\
\gamma_{\eta \zeta}^{(0)}
\end{array}\right)=\left(\begin{array}{c}
\frac{1}{A} \frac{\partial w_{0}}{\partial x}+\phi_{x} \\
\frac{\partial w_{0}}{\partial \eta}+\phi_{\eta}
\end{array}\right),
\end{aligned}
$$

where $R_{x \eta}$ is the radius of the twist, which can be derived as [31]

$$
\frac{1}{R_{x \eta}}=-\frac{k}{A^{2}} .
$$

The nonlinear parts, which are underlined in (11), will be ignored in the free vibration analysis of the blade.

The stress-strain equations can be written as

$$
\left(\begin{array}{c}
\sigma_{x} \\
\sigma_{\eta} \\
\sigma_{x \zeta} \\
\sigma_{\eta \zeta} \\
\sigma_{x \eta}
\end{array}\right)=\left[\begin{array}{ccccc}
Q_{11}(\zeta) & Q_{12}(\zeta) & 0 & 0 & 0 \\
Q_{21}(\zeta) & Q_{22}(\zeta) & 0 & 0 & 0 \\
0 & 0 & Q_{44}(\zeta) & 0 & 0 \\
0 & 0 & 0 & Q_{55}(\zeta) & 0 \\
0 & 0 & 0 & 0 & Q_{66}(\zeta)
\end{array}\right]\left(\begin{array}{c}
\varepsilon_{x} \\
\varepsilon_{\eta} \\
\gamma_{x \zeta} \\
\gamma_{\eta \zeta} \\
\gamma_{x \eta}
\end{array}\right),
$$

where the elastic constants $Q_{i j}(\zeta)$ are functions of thickness $\zeta$, which is defined as

$$
\begin{aligned}
& Q_{11}(\zeta)=Q_{22}(\zeta)=\frac{E(\zeta)}{1-\mu^{2}(\zeta)}, \\
& Q_{12}(\zeta)=Q_{21}(\zeta)=\frac{\mu(\zeta) E(\zeta)}{1-\mu^{2}(\zeta)}, \\
& Q_{44}(\zeta)=Q_{55}(\zeta)=\frac{K_{s} E(\zeta)}{2(1+\mu(\zeta))}, \\
& Q_{66}(\zeta)=\frac{E(\zeta)}{2(1+\mu(\zeta))},
\end{aligned}
$$

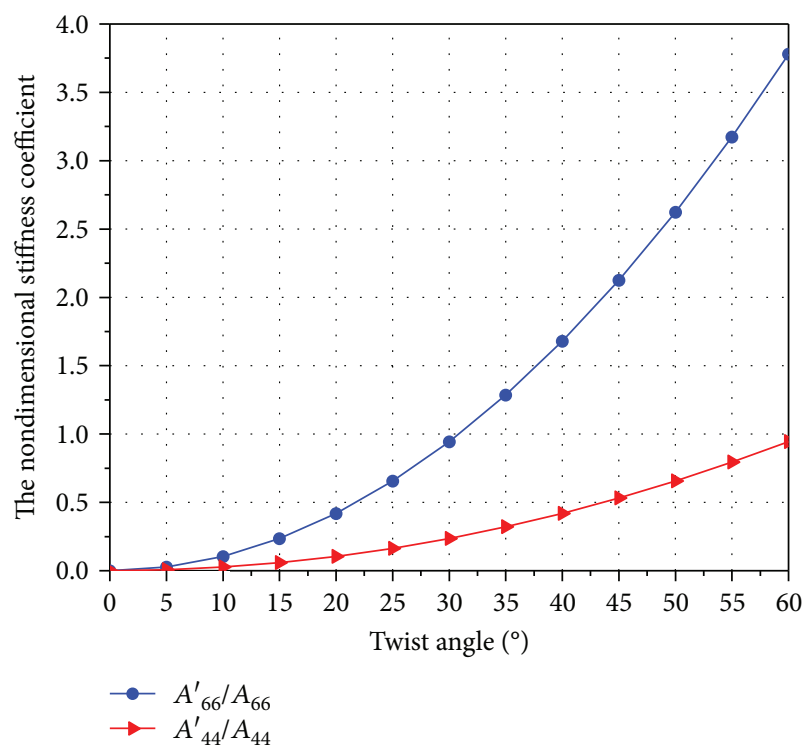

FIGURE 5: Variations of nondimensional stiffness coefficients with the initial twist angle $(\eta=0)$.

where $K_{\mathrm{s}}$ is the shear correction coefficient. Since the shear correction coefficient depends on the blade material properties and boundary conditions, it is difficult to obtain the exact value. In this paper, the shear correction coefficient $K_{\mathrm{s}}$ will be selected uniformly as 5/6 [32].

The strain energy of the blade is defined as

$$
\begin{aligned}
U_{S}= & \frac{1}{2} \int_{0}^{L} \int_{-b / 2}^{b / 2} \int_{-h / 2}^{h / 2}\left(\sigma_{x} \varepsilon_{x}+\sigma_{\eta} \varepsilon_{\eta}+\sigma_{x \zeta} \gamma_{x \zeta}+\sigma_{\eta \zeta} \gamma_{\eta \zeta}+\sigma_{x \eta} \gamma_{x \eta}\right) A d \zeta d \eta d x \\
= & \int_{0}^{L} \int_{-b / 2}^{b / 2}\left[\frac{1}{2}\left(D_{11} \kappa_{x}^{(1)^{2}}+D_{22} \kappa_{\eta}^{(1)^{2}}+D_{66} \kappa_{x \eta}^{(1)^{2}}+2 D_{12} \kappa_{x}^{(1)} \kappa_{\eta}^{(1)}\right)\right. \\
& +B_{11} \varepsilon_{x}^{(0)} \kappa_{x}^{(1)}+B_{22} \varepsilon_{\eta}^{(0)} \kappa_{\eta}^{(1)}+B_{66} \gamma_{x \eta}^{(0)} \kappa_{x \eta}^{(1)}+B_{12} \varepsilon_{x}^{(0)} \kappa_{\eta}^{(1)}+B_{21} \varepsilon_{\eta}^{(0)} \kappa_{x}^{(1)} \\
& +\frac{1}{2}\left(A_{11} \varepsilon_{x}^{(0)^{2}}+A_{22} \varepsilon_{\eta}^{(0)^{2}}+A_{66} \gamma_{x \eta}^{(0)^{2}}+2 A_{12} \varepsilon_{x}^{(0)} \varepsilon_{\eta}^{(0)}+A_{44} \gamma_{x \zeta}^{(0)^{2}}\right. \\
& \left.+A_{55} \gamma_{\eta \zeta}^{(0)^{2}}\right)+B_{66}^{\prime} w_{0} \gamma_{x \eta}^{(0)}+B_{44}^{\prime} v_{0} \gamma_{x \zeta}^{(0)}+B_{55}^{\prime} u_{0} \gamma_{\eta \zeta}^{(0)}+A_{66}^{\prime} w_{0}^{2} \\
& \left.+A_{44}^{\prime} v_{0}^{2}+A_{55}^{\prime} u_{0}^{2}\right] A d \eta d x,
\end{aligned}
$$

where $A_{i j}, B_{i j}$, and $D_{i j}$ are the extensional, bending-extensional coupling, and bending stiffness [32], $A_{i j}^{\prime}$ and $B_{i j}^{\prime}$ are the stiffness due to the initial twist of the blade. They are defined as

$$
\begin{aligned}
\left(\begin{array}{c}
A_{i j} \\
B_{i j} \\
D_{i j}
\end{array}\right) & =\int_{-h / 2}^{h / 2} Q_{i j}(\zeta)\left(\begin{array}{c}
1 \\
\zeta \\
\zeta^{2}
\end{array}\right) d \zeta \\
& =\sum_{m=1}^{3} \int_{h_{m-1}}^{h_{m}} Q_{i j}(\zeta)\left(\begin{array}{c}
1 \\
\zeta \\
\zeta^{2}
\end{array}\right) d \zeta \quad(i, j=1,2,6),
\end{aligned}
$$


TABLE 1: Convergence of nondimensional frequency parameters for the first six modes of the homogeneous material blade $(\bar{l}=3, \bar{h}=20$, and $\theta=15^{\circ}$ ).

\begin{tabular}{lccccccccccc}
\hline \multirow{2}{*}{ Mode } & \multirow{2}{*}{ ANSYS } & \multicolumn{2}{c}{$5 \times 5 \times 5$} & \multicolumn{2}{c}{$5 \times 6 \times 6$} & \multicolumn{2}{c}{$5 \times 7 \times 7$} & \multicolumn{2}{c}{$5 \times 8 \times 8$} & \multicolumn{2}{c}{$5 \times 9 \times 9$} \\
& & Value & Error & Value & Error & Value & Error & Value & Error & Value & Error \\
\hline 1st & 3.4225 & 3.4217 & $-0.02 \%$ & 3.4102 & $-0.36 \%$ & 3.4077 & $-0.43 \%$ & 3.4194 & $-0.09 \%$ & 3.4124 & $-0.30 \%$ \\
2nd & 20.7177 & 20.7606 & $0.21 \%$ & 20.7156 & $-0.01 \%$ & 20.7222 & $0.02 \%$ & 20.7540 & $0.18 \%$ & 20.7270 & $0.04 \%$ \\
3rd & 21.9608 & 22.2650 & $1.39 \%$ & 21.9718 & $0.05 \%$ & 22.1278 & $0.76 \%$ & 22.0861 & $0.57 \%$ & 22.0340 & $0.33 \%$ \\
4th & 58.8524 & 59.6322 & $1.33 \%$ & 54.3477 & $-7.65 \%$ & 58.1334 & $-1.22 \%$ & 58.1917 & $-1.12 \%$ & 58.0295 & $-1.40 \%$ \\
5th & 64.5829 & 66.5017 & $2.97 \%$ & 64.0481 & $-0.83 \%$ & 65.6567 & $1.66 \%$ & 65.4872 & $1.40 \%$ & 65.6406 & $1.64 \%$ \\
6th & 68.0114 & 68.9640 & $1.40 \%$ & 68.1982 & $0.27 \%$ & 68.6168 & $0.89 \%$ & 68.4144 & $0.59 \%$ & 68.3678 & $0.52 \%$ \\
\hline
\end{tabular}

TABLE 2: Convergence of nondimensional frequency parameters for the first six modes of the FG sandwich blade $\left(\bar{l}=3, \bar{h}=20, n=2, \bar{h}_{l}=1 / 2\right.$, and $\theta=15^{\circ}$ ).

\begin{tabular}{lcccccccccccc}
\hline \multirow{2}{*}{ Mode } & \multirow{2}{*}{ ANSYS } & \multicolumn{2}{c}{$5 \times 5 \times 5$} & \multicolumn{2}{c}{$5 \times 6 \times 6$} & \multicolumn{2}{c}{$5 \times 7 \times 7$} & \multicolumn{2}{c}{$5 \times 8 \times 8$} & \multicolumn{2}{c}{$5 \times 9 \times 9$} \\
& & Value & Error & Value & Error & Value & Error & Value & Error & Value & Error \\
\hline 1st & 4.5272 & 4.5320 & $0.11 \%$ & 4.5209 & $-0.14 \%$ & 4.5228 & $-0.10 \%$ & 4.5211 & $-0.13 \%$ & 4.5215 & $-0.13 \%$ \\
2nd & 27.3966 & 27.5019 & $0.38 \%$ & 27.4741 & $0.28 \%$ & 27.4639 & $0.25 \%$ & 27.4684 & $0.26 \%$ & 27.4665 & $0.26 \%$ \\
3rd & 29.0406 & 29.5393 & $1.72 \%$ & 29.3030 & $0.90 \%$ & 29.3432 & $1.04 \%$ & 29.2158 & $0.60 \%$ & 29.1542 & $0.39 \%$ \\
4th & 76.2376 & 75.8763 & $-0.47 \%$ & 70.8471 & $-7.07 \%$ & 74.8356 & $-1.84 \%$ & 74.8922 & $-1.76 \%$ & 75.0109 & $-1.61 \%$ \\
5th & 80.3331 & 84.3843 & $5.04 \%$ & 78.8139 & $-1.89 \%$ & 82.1073 & $2.21 \%$ & 82.2715 & $2.41 \%$ & 82.2392 & $2.37 \%$ \\
6th & 89.9847 & 91.5581 & $1.75 \%$ & 90.9359 & $1.06 \%$ & 91.0424 & $1.18 \%$ & 90.7128 & $0.81 \%$ & 90.5917 & $0.67 \%$ \\
\hline
\end{tabular}

TABLE 3: Comparison of nondimensional frequency parameters for the first six modes of homogeneous material blade $(\bar{h}=20, \mu=0.3)$.

\begin{tabular}{|c|c|c|c|c|c|c|c|c|c|c|c|c|c|}
\hline \multirow{2}{*}{$\theta$} & \multirow{2}{*}{ od } & \multicolumn{6}{|c|}{$\bar{l}=3$} & \multicolumn{6}{|c|}{$\bar{l}=1$} \\
\hline & & st & 2nd & $3 \mathrm{rd}$ & 4 th & 5 th & 6 th & 1 st & 2nd & $3 \mathrm{rd}$ & 4 th & 5 th & 6 th \\
\hline \multirow{3}{*}{$0^{\circ}$} & $\mathrm{P}$ & 3.4205 & 0.8483 & 1.3316 & 59.6783 & .2910 & 65.4353 & .4757 & .3462 & 20.9625 & 26.6650 & 0.1067 & 43.5409 \\
\hline & ANSYS & 3.4223 & 20.8230 & 21.3590 & 59.8793 & 2.2508 & 65.3690 & 3.4814 & 8.3793 & 21.0993 & .7267 & 30.2352 & 3.5819 \\
\hline & D & 140 & 00 & 21.3600 & 00 & 00 & 00 & 520 & 70 & .2900 & 700 & 800 & 43.5200 \\
\hline \multirow{3}{*}{$15^{\circ}$} & & 3.4194 & 20.7540 & 22.0861 & 58.1917 & 65.4872 & 68.4144 & 3.4479 & 10.4233 & 20.4526 & 26.6975 & 336 & 44.6423 \\
\hline & ANSYS & 3. & 77 & 21.9 & & & 14 & & & 68 & 264 & 218 & 44.4679 \\
\hline & $\operatorname{Ref}[15]$ & 3.4150 & 5700 & 22.2800 & 58.1500 & 2900 & 200 & 50 & 10.4000 & 5700 & 3500 & 32.1100 & 44.5800 \\
\hline \multirow{3}{*}{$30^{\circ}$} & Present & 3.4035 & 19.2320 & 25.4184 & 54.4781 & 70.6496 & 76.3746 & 3.4030 & 14.8939 & 18.9948 & 27.1437 & 35.2177 & 46.9877 \\
\hline & ANSYS & 3.4 & 18.9950 & 25.0621 & 55.7643 & 71.6994 & 75.9291 & 794 & & 18.4374 & 34 & 34 & 45.9808 \\
\hline & Ref [15] & 3.4170 & 19.0000 & 25.1500 & 55.9500 & 71.4000 & 76.5700 & 3.3970 & 14.3300 & 18.6900 & 27.3900 & 34.9000 & 46.5200 \\
\hline \multirow{3}{*}{$45^{\circ}$} & Present & 3.4163 & 17.4172 & 29.7580 & 54.0239 & 80.8963 & 87.6963 & 3.3387 & 20.0628 & 17.1876 & 28.2967 & 40.1453 & 48.6332 \\
\hline & & & & & & & & & & & & 37.3254 & 46.1459 \\
\hline & $\operatorname{Ref}[15]$ & 3.4200 & 16.9900 & 29.2000 & 53.5800 & 78.8200 & 87.0500 & 3.3230 & 18.3200 & 16.3900 & 27.7100 & 38.1700 & 47.3000 \\
\hline
\end{tabular}

$$
\begin{aligned}
A_{i j} & =\int_{-h / 2}^{h / 2} Q_{i j}(\zeta) d \zeta \\
& =\sum_{m=1}^{3} \int_{h_{m-1}}^{h_{m}} Q_{i j}(\zeta) d \zeta \quad(i, j=4,5), \\
B_{i j}^{\prime} & =\left\{\begin{array}{cc}
-\frac{1}{R_{x \eta}} B_{i j} & (i=j=4,5), \\
\frac{2}{R_{x \eta}} B_{i j} & (i=j=6),
\end{array}\right.
\end{aligned}
$$$$
A_{i j}^{\prime}= \begin{cases}\frac{1}{2 R_{x \eta}^{2}} A_{i j} & (i=j=4,5), \\ \frac{2}{R_{x \eta}^{2}} A_{i j} & (i=j=6) .\end{cases}
$$

It should be noted that the stiffness $B_{i j}$ and $B_{i j}^{\prime}$ will be zero while the structure is symmetrical about the middle surface in the thickness direction. Figure 5 shows the variations of the 
nondimensional stiffness coefficients $\left(A_{44}^{\prime} / A_{44}\right.$ and $A_{66}^{\prime} / A_{66}$ at $\eta=0)$ with the initial twist angle. It can be found that both the nondimensional stiffness coefficients increase with increase in the initial twist angle.

2.3. The Potential Energy of Centrifugal Force. The coordinate system transformation relationship between the $x y z$-coordinate and $X Y Z$-coordinate is given by

$$
\left(\begin{array}{c}
\mathbf{i}_{x} \\
\mathbf{i}_{y} \\
\mathbf{i}_{z}
\end{array}\right)=\left[\begin{array}{ccc}
1 & 0 & 0 \\
0 & \cos \varphi & \sin \varphi \\
0 & -\sin \varphi & \cos \varphi
\end{array}\right]\left(\begin{array}{c}
\mathbf{i}_{X} \\
\mathbf{i}_{Y} \\
\mathbf{i}_{Z}
\end{array}\right) .
$$

Then, the angular velocity $\boldsymbol{\omega}$ in the $x y z$-coordinate of the pretwisted plate is expressed as

$$
\boldsymbol{\omega}=\left[\begin{array}{c}
0 \\
\Omega \cos \varphi \\
-\Omega \sin \varphi
\end{array}\right]^{T}\left(\begin{array}{l}
\mathbf{i}_{x} \\
\mathbf{i}_{y} \\
\mathbf{i}_{z}
\end{array}\right) .
$$

The centrifugal force $\mathbf{F}_{\mathrm{C}}$ per unit volume in the blade is given by

$$
\begin{aligned}
\mathbf{F}_{\mathrm{C}} & =\rho \boldsymbol{\omega} \times\left(\mathbf{r}^{(0)}+R \mathbf{i}_{x}\right) \times \boldsymbol{\omega} \\
R+x-k \eta \frac{\zeta}{A} & {\left[\begin{array}{c} 
\\
\eta \sin \Phi \sin \varphi+\zeta \cos \Phi \sin \frac{\varphi}{A} \\
\eta \sin \Phi \cos \varphi+\zeta \cos \Phi \cos \frac{\varphi}{A}
\end{array}\right]^{2}\left(\begin{array}{c}
\mathbf{i}_{x} \\
\mathbf{i}_{y} \\
\mathbf{i}_{z}
\end{array}\right), }
\end{aligned}
$$

where $\Phi$ is the sum of the stagger angle and the twist angle of the point, given by

$$
\Phi=\varphi+\theta^{\prime}
$$

The rotation of the blade about the turbine axial direction produces a significant amount of membrane stresses, which are essentially acting in the radial direction (the spanwise and chordwise directions of the blade). The component of centrifugal force along the spanwise direction $\sigma_{\mathrm{cs}}$ and the chordwise direction $\sigma_{c c}$ can be written as $[10,14]$

$$
\begin{aligned}
\sigma_{\mathrm{cs}}= & \int_{X}^{L} \mathbf{F}_{\mathrm{C}} \cdot \mathbf{e}_{0 x} A d x=\rho \Omega^{2}\left\{\left(R-\frac{1}{2 A} k \eta \zeta\right)(L-x)\right. \\
& +\frac{1}{2}\left(L^{2}-x^{2}\right)+\frac{1}{4} \eta^{2}(\cos 2 \Phi-\cos 2 \Theta) \\
& \left.+\frac{1}{4 A} \eta \zeta(\sin 2 \Phi-\sin 2 \Theta)\right\},
\end{aligned}
$$

TABLe 4: The materials and properties of the FG sandwich blade.

\begin{tabular}{lcl}
\hline Materials & \multicolumn{2}{c}{ Material properties } \\
\hline $\begin{array}{l}\text { Metal } \\
\text { (alloy C-276) }\end{array}$ & $E_{m}=2.08 \times 10^{11} \mathrm{~Pa}, \quad \mu_{m}=0.32, \quad \rho_{m}=8900 \mathrm{~kg} / \mathrm{m}^{3}$ \\
$\begin{array}{l}\text { Ceramic } \\
\left(\mathrm{Al}_{2} \mathrm{O}_{3}\right)\end{array}$ & $E_{c}=3.50 \times 10^{11} \mathrm{~Pa}, \quad \mu_{c}=0.26, \quad \rho_{c}=3970 \mathrm{~kg} / \mathrm{m}^{3}$ \\
\hline
\end{tabular}

$$
\begin{aligned}
\sigma_{c c} & =\int_{b / 2}^{0} \mathbf{F}_{\mathrm{C}} \cdot \mathbf{e}_{0 \eta} d \eta \\
& =\rho \Omega^{2}\left\{\frac{1}{2 k} \zeta \sin 2 \Phi \sinh ^{-1}\left(\frac{1}{2} k b\right)-\frac{1}{8} b^{2} \sin ^{2} \Phi\right\},
\end{aligned}
$$

where $\Theta$ is the sum of the stagger angle and the total twist angle, given by

$$
\Theta=\varphi+\theta
$$

The centrifugal potential energy can be derived as

$$
U_{\mathrm{CF}}=\frac{1}{2} \int_{0}^{L} \int_{-b / 2}^{b / 2} \int_{-h / 2}^{h / 2}\left(\sigma_{\mathrm{cs}} \varepsilon_{\mathrm{cs}}+\sigma_{\mathrm{cc}} \varepsilon_{\mathrm{cc}}\right) A d \zeta d \eta d x \text {. }
$$

According to [26], $\varepsilon_{\mathrm{cs}}$ and $\varepsilon_{\mathrm{cc}}$ are given by

$$
\begin{aligned}
& \varepsilon_{\mathrm{cs}}=\left(\frac{\partial v_{0}}{\partial x}\right)^{2}+\left(\frac{\partial w_{0}}{\partial x}\right)^{2}, \\
& \varepsilon_{\mathrm{cc}}=\left(\frac{\partial u_{0}}{\partial \eta}\right)^{2}+\left(\frac{\partial w_{0}}{\partial \eta}\right)^{2} .
\end{aligned}
$$

Substituting (21) and (24) into (23), the potential energy can be written as

$$
\begin{aligned}
U_{\mathrm{CF}}= & \int_{0}^{L} \int_{-b / 2}^{b / 2}\left\{\left(\frac{I_{1}}{8} b \sin 2 \Phi-\frac{I_{0}}{16} b^{2} \sin ^{2} 2 \Phi\right)\right. \\
& \cdot\left[\left(\frac{\partial u_{0}}{\partial \eta}\right)^{2}+\left(\frac{\partial w_{0}}{\partial \eta}\right)^{2}\right] \\
& +I_{1}\left[\frac{\eta}{8 A}(\sin 2 \Phi-\sin 2 \Theta)-\frac{k \eta(L-x)}{4 A}\right] \\
& \cdot\left[\left(\frac{\partial v_{0}}{\partial x}\right)^{2}+\left(\frac{\partial w_{0}}{\partial x}\right)^{2}\right] \\
& +I_{0}\left[\frac{1}{2} R(L-x)+\frac{1}{4}\left(L^{2}-x^{2}\right)+\frac{\eta^{2}}{8}(\cos 2 \Phi-\cos 2 \Theta)\right] \\
& \left.\cdot\left[\left(\frac{\partial v_{0}}{\partial x}\right)^{2}+\left(\frac{\partial w_{0}}{\partial x}\right)^{2}\right]\right\} A d \eta d x
\end{aligned}
$$

The total potential energy can be written as

$$
U=U_{\mathrm{S}}+U_{\mathrm{CF}}
$$


TABLE 5: Comparisons of nondimensional frequency parameters for the first six modes of the FG sandwich blade $(\bar{l}=3, \bar{h}=20, n=2$, and $\left.\bar{h}_{l}=1 / 2\right)$.

\begin{tabular}{|c|c|c|c|c|c|c|c|c|c|c|c|c|}
\hline \multirow{2}{*}{ Mode } & \multicolumn{4}{|c|}{$0^{\circ}$} & \multicolumn{4}{|c|}{$15^{\circ}$} & \multicolumn{4}{|c|}{$30^{\circ}$} \\
\hline & Present & ANSYS & Error & Mode shape & Present & ANSYS & Error & Mode shape & Present & ANSYS & Error & Mode shape \\
\hline 1 st & 4.5233 & 4.5270 & $-0.08 \%$ & $1 \mathrm{~B}$ & 4.5206 & 4.5272 & $-0.15 \%$ & $1 \mathrm{~B}$ & 4.4729 & 4.5290 & $-1.24 \%$ & $1 \mathrm{~B}$ \\
\hline 2nd & 27.9633 & 27.7729 & $0.69 \%$ & $1 \mathrm{~T}$ & 27.4621 & 27.3966 & $0.24 \%$ & $2 \mathrm{~B}$ & 25.4167 & 25.0992 & $1.26 \%$ & $2 \mathrm{~B}$ \\
\hline $3 \mathrm{rd}$ & 28.3106 & 28.2605 & $0.18 \%$ & $2 \mathrm{~B}$ & 29.1565 & 29.0406 & $0.40 \%$ & $1 \mathrm{~T}$ & 33.0633 & 32.5549 & $1.56 \%$ & $1 \mathrm{~T}$ \\
\hline 4 th & 75.9386 & 75.8281 & $0.15 \%$ & $1 \mathrm{~EB}$ & 74.8841 & 76.2376 & $-1.78 \%$ & $3 \mathrm{~B}$ & 73.0937 & 72.9145 & $0.25 \%$ & $3 \mathrm{~B}$ \\
\hline 5 th & 79.3035 & 79.1454 & $0.20 \%$ & $3 \mathrm{~B}$ & 82.0284 & 80.3331 & $2.11 \%$ & $1 \mathrm{~EB}$ & 89.2792 & 88.7755 & $0.57 \%$ & $1 \mathrm{~EB}$ \\
\hline 6th & 87.5852 & 87.0515 & $0.61 \%$ & $2 \mathrm{~T}$ & 90.5827 & 89.9847 & $0.66 \%$ & $2 \mathrm{~T}$ & 99.5237 & 98.9166 & $0.61 \%$ & $2 \mathrm{~T}$ \\
\hline
\end{tabular}

2.4. The Kinetic Energy. The displacement vector u can be written as

$$
\begin{aligned}
\mathbf{u} & =[u v w]\left(\begin{array}{c}
\mathbf{e}_{0 x} \\
\mathbf{e}_{0 \eta} \\
\mathbf{e}_{0 \zeta}
\end{array}\right) \\
& =\frac{1}{A}\left[\begin{array}{c}
u-k \eta w \\
A v \cos \theta^{\prime}-(k \eta u+w) \sin \theta^{\prime} \\
A v \sin \theta^{\prime}+(k \eta u+w) \cos \theta^{\prime}
\end{array}\right]^{T}\left(\begin{array}{c}
\mathbf{i}_{x} \\
\mathbf{i}_{y} \\
\mathbf{i}_{z}
\end{array}\right) .
\end{aligned}
$$

An arbitrary point of the blade after deformation can be expressed by the position vector $\mathbf{r}$ as

$$
\begin{aligned}
\mathbf{r} & =\mathbf{r}^{(0)}+\mathbf{u} \\
& =\frac{1}{A}\left[\begin{array}{c}
u+A x-k \eta(w+\zeta) \\
A(v+\eta) \cos \theta^{\prime}-(k \eta u+w+\zeta) \sin \theta^{\prime} \\
A(v+\eta) \sin \theta^{\prime}+(k \eta u+w+\zeta) \cos \theta^{\prime}
\end{array}\right]^{T}\left(\begin{array}{c}
\mathbf{i}_{x} \\
\mathbf{i}_{y} \\
\mathbf{i}_{z}
\end{array}\right) .
\end{aligned}
$$

The velocity $\mathbf{V}_{\mathrm{r}}$ of the point due to the rotation can be derived as

$$
\begin{aligned}
\mathbf{V}_{\mathrm{r}} & =\boldsymbol{\omega} \times\left(\mathbf{r}+R \mathbf{i}_{x}\right) \\
& =\frac{\Omega}{A}\left[\begin{array}{c}
A(v+\eta) \sin \Phi+(k \eta u+w+\zeta) \cos \Phi \\
{[k \eta(w+\zeta)-A(R+x)-u] \sin \varphi} \\
{[k \eta(w+\zeta)-A(R+x)-u] \cos \varphi}
\end{array}\right]^{T}\left(\begin{array}{c}
\mathbf{i}_{x} \\
\mathbf{i}_{y} \\
\mathbf{i}_{z}
\end{array}\right)
\end{aligned}
$$

The kinetic energy reads

$$
\begin{aligned}
T= & \frac{1}{2} \int_{0}^{L} \int_{-b / 2}^{b / 2} \int_{-h / 2}^{h / 2} \rho\left[\left(\dot{u}+\mathbf{V}_{\mathrm{r}} \cdot \mathbf{e}_{0 x}\right)^{2}+\left(\dot{v}+\mathbf{V}_{\mathrm{r}} \cdot \mathbf{e}_{0 \eta}\right)^{2}\right. \\
& \left.+\left(\dot{w}+\mathbf{V}_{\mathrm{r}} \cdot \mathbf{e}_{0 \zeta}\right)^{2}\right] A d \zeta d \eta d x=T_{i n}+T_{g y}+T_{r o}
\end{aligned}
$$

where

$$
\begin{aligned}
T_{i n}= & \int_{0}^{L} \int_{-b / 2}^{b / 2}\left[\frac{1}{2} I_{2}\left(\dot{\phi}_{x}^{2}+\dot{\phi}_{\eta}^{2}\right)+I_{1}\left(\dot{u}_{0} \dot{\phi}_{x}+\dot{v}_{0} \dot{\phi}_{\eta}\right)\right. \\
& \left.+\frac{1}{2} I_{0}\left(\dot{u}_{0}^{2}+\dot{v}_{0}^{2}+\dot{w}_{0}^{2}\right)\right] A d \eta d x \\
T_{g y}= & \Omega \int_{0}^{L} \int_{-b / 2}^{b / 2}\left\{\left(\phi_{\eta} \sin \Phi+A \cos \Phi\right)\left(I_{2} \dot{\phi}_{x}+I_{1} \dot{u}_{0}\right)\right. \\
& +\left(k \eta-\phi_{x}\right)\left(I_{2} \dot{\phi}_{\eta}+I_{1} \dot{v}_{0}\right) \sin \Phi+\left[\left(\eta+v_{0}\right) \sin \Phi\right. \\
& \left.+\left(A w_{0}-k \eta(R+x)\right) \cos \Phi\right]\left(I_{1} \dot{\phi}_{x}+I_{0} \dot{u}_{0}\right) \\
& +\left(k \eta w_{0}-A R-A x-u_{0}\right)\left(I_{1} \dot{\phi}_{\eta}+I_{0} \dot{v}_{0}\right) \sin \Phi \\
& -I_{0}\left[\left(k \eta^{2}+k \eta v_{0}\right) \sin \Phi+\left(A u_{0}+R+x\right) \cos \Phi\right] \dot{w}_{0} \\
& \left.-I_{1}\left(A \varphi_{x} \cos \Phi+k \eta \varphi_{\eta} \sin \Phi\right) \dot{w}_{0}\right\} d \eta d x,
\end{aligned}
$$

$$
\begin{aligned}
T_{r o}= & \Omega^{2} \int_{0}^{L} \int_{-b / 2}^{b / 2}\left\{\frac{1}{2}\left(I_{2}+I_{0}\right)\left(\phi_{\eta} \sin \Phi+\frac{1}{A}\left(1+k \eta \phi_{x}\right) \cos \Phi\right)^{2}\right. \\
& +\frac{1}{2 A^{2}} I_{2}\left(\phi_{x}-k \eta\right)^{2}+\frac{1}{2} I_{0}\left(R+x+\frac{1}{A} u_{0}-\frac{1}{A} k \eta w_{0}\right)^{2} \\
& +I_{1}\left[\left(\eta+v_{0}\right) \sin \Phi+\frac{1}{A}\left(w_{0}+k \eta u_{0}\right) \cos \Phi\right] \\
& \cdot\left(\phi_{\eta} \sin \Phi+\frac{1}{A}\left(1+k \eta \phi_{x}\right) \cos \Phi\right) \\
& \left.+\frac{1}{A^{2}} I_{1}\left[A(R+x)+u_{0}-k \eta w_{0}\right]\left(\phi_{x}-k \eta\right)\right\} A d \eta d x
\end{aligned}
$$

where "." is the first-order derivation of time; $I_{0}, I_{1}$, and $I_{2}$ in (25) and (30) are defined in terms of the density $\rho(\zeta)$ as 


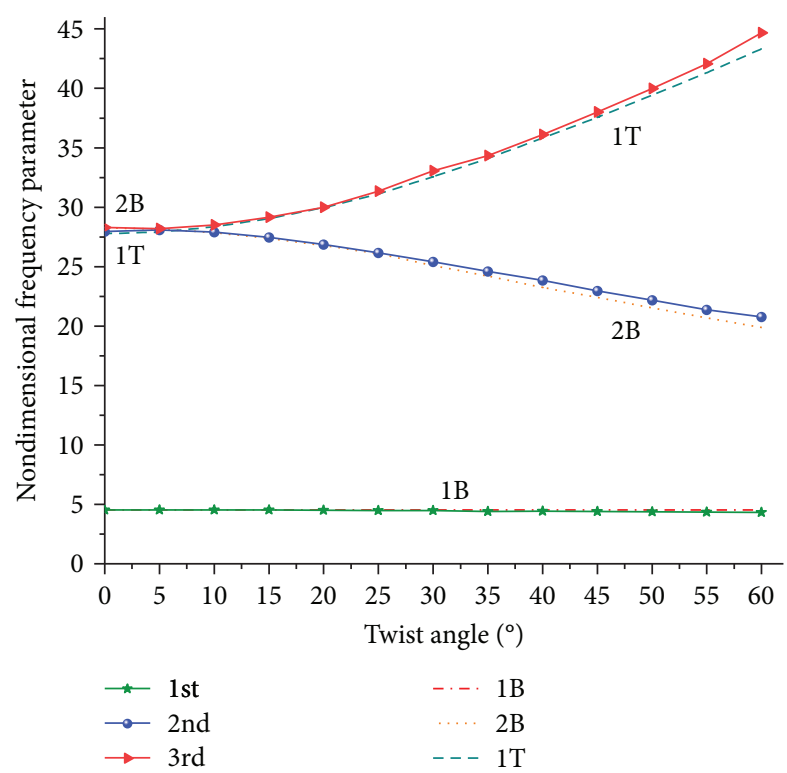

(a) 1st to 3rd modes

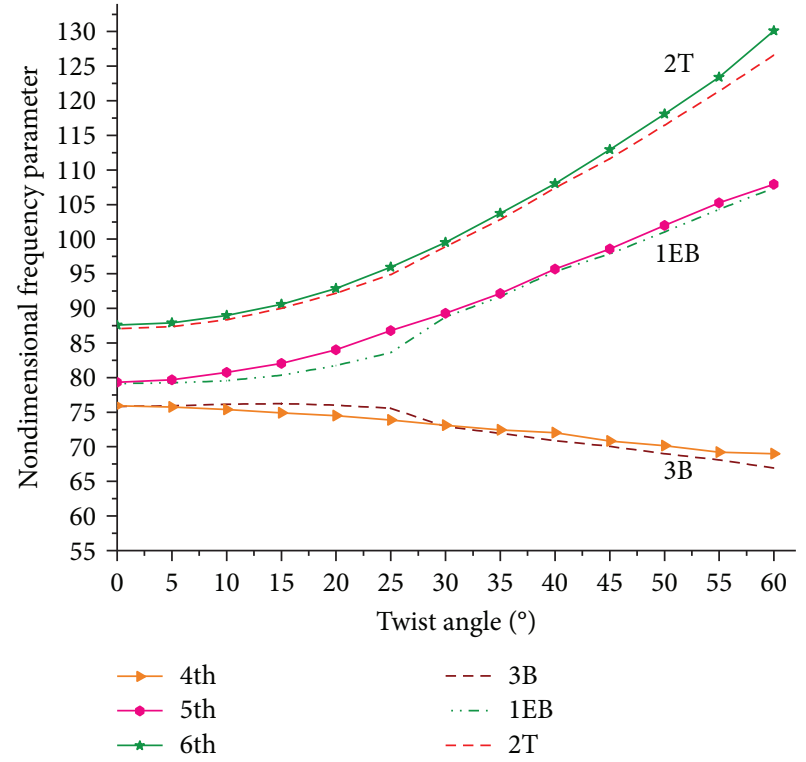

(b) 4th to 6th modes

FIGURE 6: Variations of nondimensional frequency parameters with the twist angle $\left(\bar{l}=3, \bar{h}=20, n=2\right.$, and $\left.\bar{h}_{l}=1 / 2\right)$.

$$
\left(\begin{array}{c}
I_{0} \\
I_{1} \\
I_{2}
\end{array}\right)=\int_{-h / 2}^{h / 2} \rho(\zeta)\left(\begin{array}{c}
1 \\
\zeta \\
\zeta^{2}
\end{array}\right) d \zeta=\sum_{m=1}^{3} \int_{h_{m-1}}^{h_{m}} \rho(\zeta)\left(\begin{array}{c}
1 \\
\zeta \\
\zeta^{2}
\end{array}\right) d \zeta
$$

\section{Frequency Solving}

The Rayleigh-Ritz method is used to solve the natural frequency of the blade; the expressions of the displacements and rotations are assumed

$$
\begin{aligned}
& u_{0}(x, \eta, t)=\sum_{N=1}^{\infty} U_{N}(x, \eta) e^{i \omega_{N} t} \\
& v_{0}(x, \eta, t)=\sum_{N=1}^{\infty} V_{N}(x, \eta) e^{i \omega_{N} t} \\
& w_{0}(x, \eta, t)=\sum_{N=1}^{\infty} W_{N}(x, \eta) e^{i \omega_{N} t} \\
& \phi_{x}(x, \eta, t)=\sum_{N=1}^{\infty} \Phi_{x N}(x, \eta) e^{i \omega_{N} t} \\
& \phi_{\eta}(x, \eta, t)=\sum_{N=1}^{\infty} \Phi_{\eta N}(x, \eta) e^{i \omega_{N} t}
\end{aligned}
$$

The functions $U_{N}(x, \eta), V_{N}(x, \eta), W_{N}(x, \eta), \Phi_{x N}(x, \eta)$, and $\Phi_{\eta N}(x, \eta)$ represent the $N$ order mode shape, and $\omega_{N}$ is the corresponding circular frequency. The algebraic polynomials can form a mathematically complete set of functions, which guarantees convergence to the exact solution as the

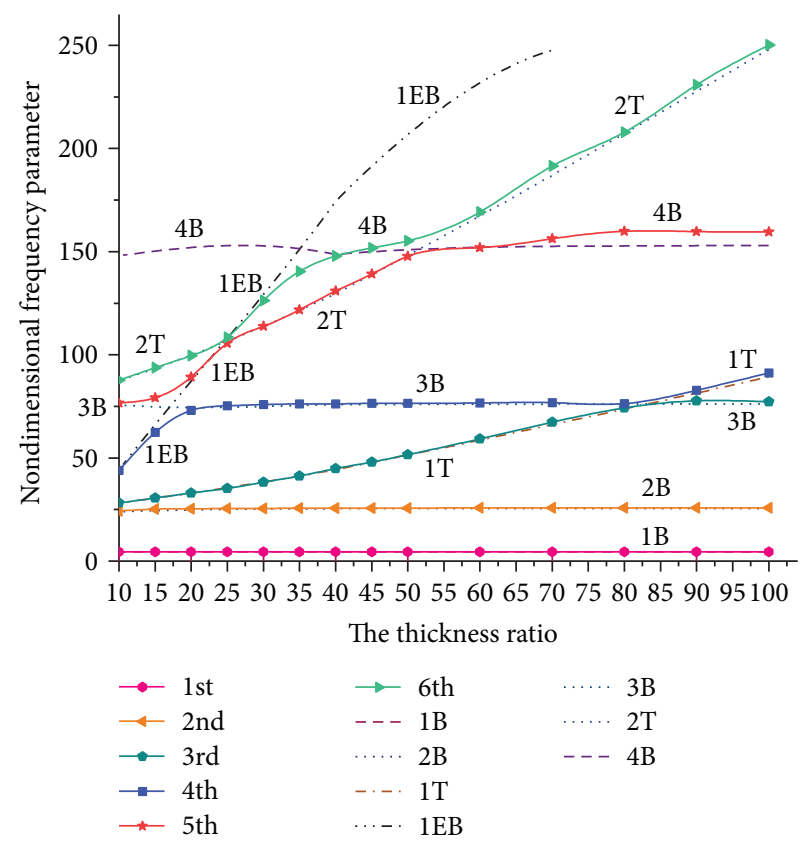

FIGURE 7: Variations of nondimensional frequency parameters with the thickness ratio $\left(\bar{l}=3, n=2, \bar{h}_{l}=1 / 2\right.$, and $\left.\theta=30^{\circ}\right)$.

number of terms taken increases [5]. Thus, in this paper, the mode shapes are expanded in terms of two-dimensional algebraic polynomials in terms of the nondimensional coordinates $\chi$ and $\xi$ as

$$
U_{N}(\chi, \xi)=\sum_{i=1}^{I} \sum_{j=0}^{J} U_{i j} \chi^{i} \xi^{j},
$$




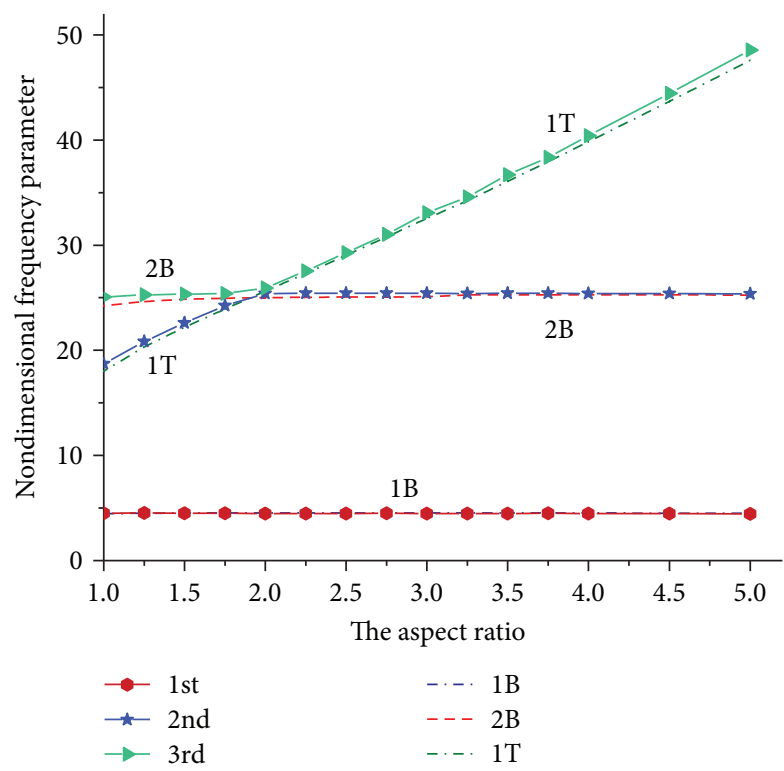

(a) 1 st to 3 rd modes

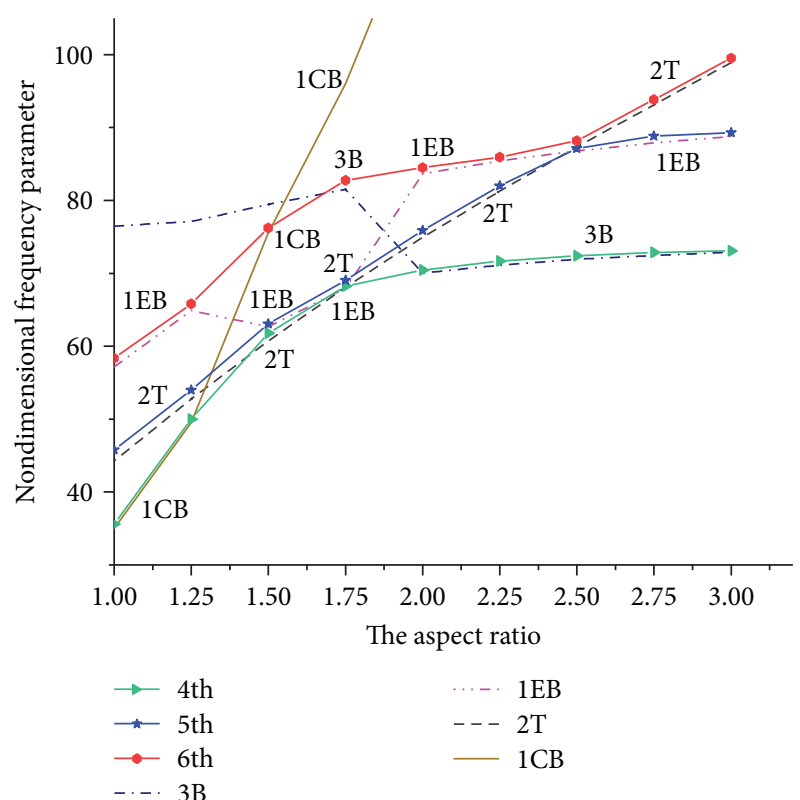

(b) 4 th to 6 th modes

FIGURE 8: Variations of nondimensional frequency parameters with the aspect ratio $\left(\bar{h}=20, n=2, \bar{h}_{l}=1 / 2, \theta=30^{\circ}\right)$.

$$
\begin{aligned}
V_{N}(\chi, \xi) & =\sum_{k=1}^{K} \sum_{l=0}^{L} V_{k l} \chi^{k} \xi^{l}, \\
W_{N}(\chi, \xi) & =\sum_{m=2}^{M} \sum_{n=0}^{N} W_{m n} \chi^{m} \xi^{n} \\
\Phi_{x N}(\chi, \xi) & =\sum_{i=1}^{I} \sum_{j=0}^{J} \Phi_{x i j} \chi^{i} \xi^{j} \\
\Phi_{\eta N}(\chi, \xi) & =\sum_{k=1}^{K} \sum_{l=0}^{L} \Phi_{\eta k l} \chi^{k} \xi^{l},
\end{aligned}
$$

where $\chi=x / L$ and $\xi=2 \eta / b . U_{i j}, V_{k l}, W_{m n}, \Phi_{x i j}$, and $\Phi_{\eta k l}$ are undetermined coefficients. The Rayleigh-Ritz method imposes a necessary condition that trial functions must satisfy the geometric boundary conditions arbitrarily. The cantilever blade is clamped at $\chi=0(u=v=w=d w / d x=0)$ along the spanwise direction and is completely free at $\chi=1$, $\xi=-1$, and $\xi=1$. The indices $i, k$, and $m$ in (33), (46), and (47) begin with $i=1, k=1$, and $m=2$; it can be ensured that all terms of polynomials satisfied the geometric boundary conditions.

To solve the free frequency of the blade, (32) and (33) are substituted into (26) and (30) in order to obtain the maximum strain energy $\left(U_{\max }\right)$ and kinetic energy $\left(T_{\max }\right)$ by setting $t=0$. The Rayleigh-Ritz method requires the minimization of the function $\Pi=T_{\max }-U_{\max }$, which can be achieved by taking the derivatives

$$
\begin{aligned}
& \frac{\partial \prod}{U_{i j}}=0, \\
& \frac{\partial \prod}{\Phi_{x_{i j}}}=0 \quad(i=1,2, \ldots, I ; j=0,1, \ldots, J), \\
& \frac{\partial \prod}{V_{k l}}=0, \\
& \frac{\partial \prod}{\Phi_{\eta_{k l}}}=0 \quad(k=1,2, \ldots, K, l=0,1, \ldots, L), \\
& \frac{\partial \prod}{W_{m n}}=0 \quad(m=2,3, \ldots, M, n=0,1, \ldots, N) .
\end{aligned}
$$

There are a total of $2 \times I \times(J+1)+2 \times K \times(L+1)+$ $(M-1) \times(N+1)$ equations, which can be described by

$$
\left\{\mathbf{K}-\omega_{N}^{2} \mathbf{M}\right\}[\Delta]=0,
$$

where $\mathbf{K}$ and $\mathbf{M}$ are the stiffness and mass matrices, respectively; $[\Delta]$ is the vector of undetermined coefficients, given by

$$
[\Delta]=\left[\begin{array}{lllll}
U_{i j} & V_{k l} & W_{m n} & \Phi_{x_{i j}} & \Phi_{\eta_{k l}}
\end{array}\right]^{T}
$$

The generalized eigenvalues (natural frequency) can be obtained by setting the coefficient matrix of (35) equal to zero, and the corresponding mode shape can be determined by substituting the eigenvector $[\Delta]$ back into (33). 


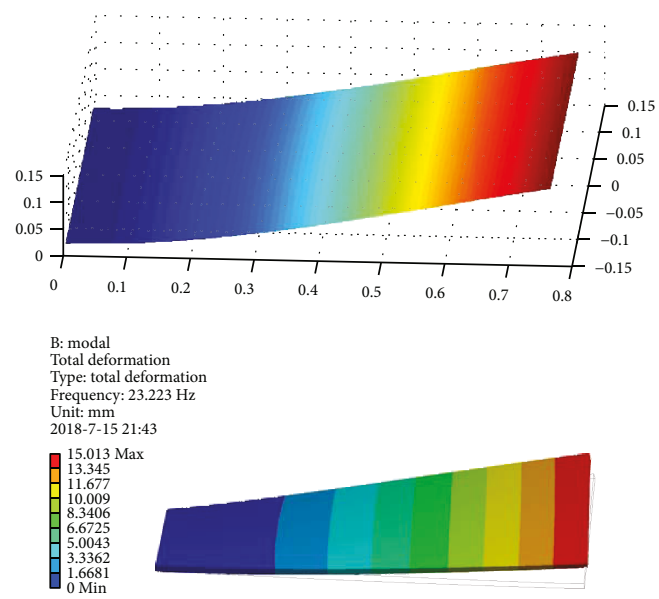

(a)

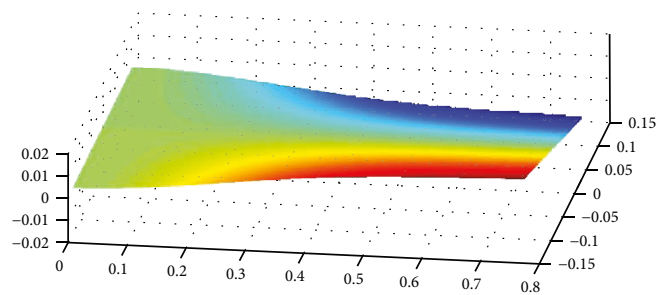

B: modal

Total deformation

Type: total deformation
Frequency: $166.93 \mathrm{~Hz}$

Unit: $\mathrm{mm}$ 2018-7-15 21:4

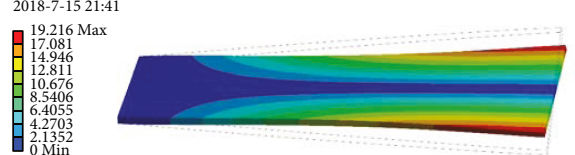

(c)

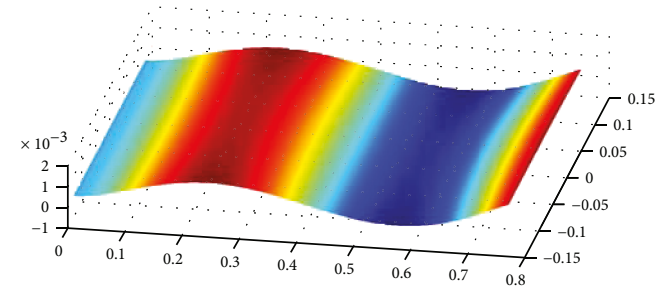

B: modal
Total deformation

Total deformation
Type: total deformation

Frequency: $455.21 \mathrm{~Hz}$

Unit: $\mathrm{mm}$
$2018-7-1521: 39$

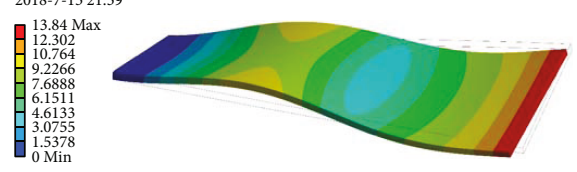

(e)

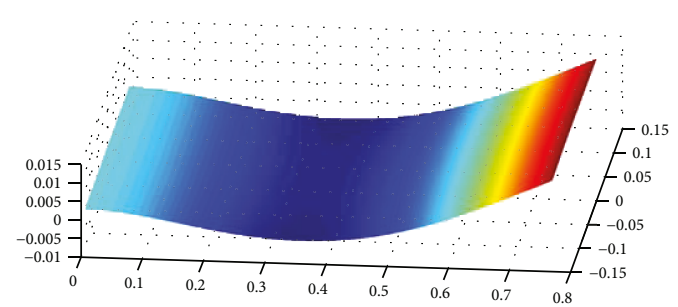

B: modal
Total deformatio

Type: total deformatio
Frequency: $128.7 \mathrm{~Hz}$

Unit: $m$ m
2018-7-15 21:42

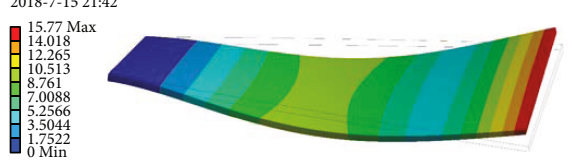

(b)

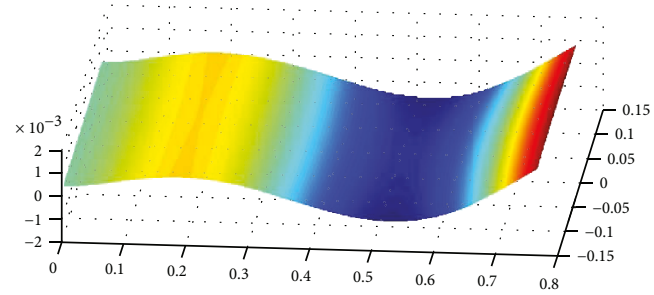

B: modal

Total deformation

Fype: total deformatio

Unit: $\mathrm{mm}$

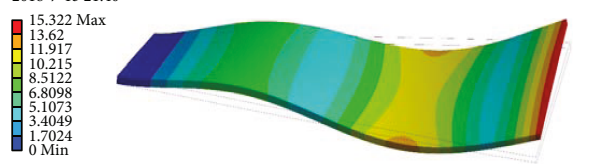

(d)

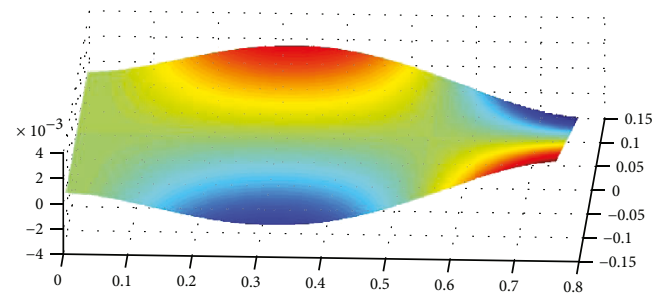

B: modal

Total deformation

Type: total deformation

Unit: $\mathrm{mm}$

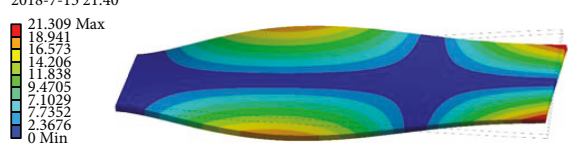

(f)

FIGURE 9: Mode shapes of the FG sandwich blade. (A1) 1st mode shape by Matlab; (B1) 2nd mode shape by Matlab; (C1) 3rd mode shape by Matlab; (D1) 4th mode shape by Matlab; (E1) 5th mode shape by Matlab; (F1) 6th mode shape by Matlab; (A2) 1st mode shape by ANSYS; (B2) 2nd mode shape by ANSYS; (C2) 3rd mode shape by ANSYS; (D2) 4th mode shape by ANSYS; (E2) 5th mode shape by ANSYS; (F2) 6th mode shape by ANSYS.

\section{Numerical Results and Analyses}

4.1. Convergence Analysis. A typical example of pretwisted blade with chord length $b=0.254 \mathrm{~m}$, aspect ratio $\bar{l}=L / b$ from 1 to 5 , thickness ratio $\bar{h}=b / h$ from 10 to 100 , twist angle $\theta$ from $0^{\circ}$ to $60^{\circ}$, layer thickness ratio $\bar{h}_{l}=\left(h-h_{m}\right) / 2 h_{m}$ from 0.25 to 4 , scalar parameter of volume fraction of FGM $n$ from 0.1 to 10 , stagger angle $\varphi$ from $0^{\circ}$ to $90^{\circ}$, and disk radius ratio $\bar{r}=R / b$ is used. 
TABLE 6: Nondimensional frequency parameters for the first six modes of FG sandwich blade with varying twist angle $(\bar{l}=3, \bar{h}=20, n=2$, and $\left.\bar{h}_{l}=1 / 2\right)$.

\begin{tabular}{lccccccccccc}
\hline Mode & $0^{\circ}$ & $5^{\circ}$ & $10^{\circ}$ & $15^{\circ}$ & $20^{\circ}$ & $25^{\circ}$ & $30^{\circ}$ & $45^{\circ}$ & $50^{\circ}$ & $60^{\circ}$ \\
\hline 1st & 4.5233 & 4.5342 & 4.5342 & 4.5206 & 4.5041 & 4.4935 & 4.4743 & 4.4020 & 4.3605 & 4.3245 \\
2nd & 27.9633 & 28.0799 & 27.9178 & 27.4621 & 26.8716 & 26.1715 & 25.4167 & 22.9643 & 22.1860 & 20.7649 \\
3rd & 28.3106 & 28.2050 & 28.5154 & 29.1565 & 29.9955 & 31.3439 & 33.0633 & 38.0040 & 39.9970 & 44.6692 \\
4th & 75.9386 & 75.7447 & 75.3807 & 74.8841 & 74.5120 & 73.8517 & 73.0937 & 70.8379 & 70.1630 & 68.9817 \\
5th & 79.3035 & 79.7009 & 80.7576 & 82.0284 & 84.0329 & 86.7774 & 89.2792 & 98.6081 & 101.9785 & 107.9219 \\
6th & 87.5852 & 87.9114 & 88.9680 & 90.5827 & 92.8839 & 95.9725 & 99.5237 & 112.9271 & 118.0741 & 130.0957 \\
\hline
\end{tabular}

All the numerical results are presented in the form of the nondimensional frequency parameter $\bar{\omega}=\omega_{N} L^{2} \sqrt{\rho h / D}=$ $\omega_{N} L^{2} / h \sqrt{12 \rho\left(1-\mu^{2}\right) / E}$ for the homogeneous material blade, where $D=E h^{3} / 12\left(1-\mu^{2}\right)$ is the blade flexural rigidity per unit length and $\bar{\omega}=\omega_{N} L^{2} / h \sqrt{12 \rho_{m}\left(1-\mu_{m}^{2}\right) / E_{m}}$ for the functionally graded sandwich blade in the following section, and the nondimensional rotation velocity is $\bar{\Omega}=\Omega / \omega_{1}$, where $\omega_{1}$ is the first static circular frequency $(\Omega=0)$ of the functionally graded sandwich blade.

According to [5], the number of terms chosen for each component of the displacements is the same. The results of the homogeneous material blade and functionally graded sandwich blade are given in Tables 1 and 2. The maximum difference between the $5 \times 9 \times 9=405$ term and the $5 \times 8 \times$ $8=320$ term solutions for the first six modes of the homogeneous material blade is no more than $0.28 \%$, and $0.21 \%$ for the FG sandwich blade. But the time spent in computing has multiplied. Moreover, the eigenvalues are more likely to be ill-conditioned with the increase in the size of the matrix in numerical calculation. Therefore, it has been decided to use $5 \times 8 \times 8=320$ terms in the subsequent analysis.

The data used for comparison below "ANSYS" in all tables are computed by the finite element model in ANSYS Workbench (version 17.2). The middle surface model of the blade is established, and the finite element analysis is performed by using the SHELL181 linear quadrilateral element type. The layered section object in ANSYS mechanical is used to define the layered section of composite modeling. In order to get accurate results as much as possible, 20 layers in each skin (total of 41 layers in the thickness direction) are defined to simulate the cross section of the functionally graded sandwich blade.

4.2. Comparative Studies. The homogeneous material blade is used first to demonstrate the numerical accuracy of the analytical model in this paper, and the first six nondimensional frequency parameters are compared with ANSYS and reference data [15] in Table 3. The results are in good agreement when the twist angle $\theta=0^{\circ}$ to $45^{\circ}$ in the case of the aspect ratio $\bar{l}=3$. The results match well also when the twist angle $\theta=0^{\circ}$ to $30^{\circ}$ in the case of the aspect ratio $\bar{l}=1$, but the maximum error (occurring at the 2 nd order) is about $8.86 \%$ when $\theta$ increases to $45^{\circ}$. The reason is that the Lame parameter $A$ is approximated to 1 in order to improve the calculation speed in the process of numerical calculation. The error is smaller in the case of a larger aspect ratio and smaller twist angle, but the error becomes larger as the aspect ratio decreases and the twist angle increases. Thus, the aspect ratio $\bar{l}=3$ is used to obtain more accurate results.

The functionally graded sandwich blade is composed of metal and ceramic materials; their properties are given in Table 4. In Table 5, the first six nondimensional frequency parameters $\bar{\omega}$ of the FG sandwich blade are compared with results in ANSYS Workbench, both showing good agreements, respectively.

The mode shapes corresponding to the first six orders of frequencies are also listed in Table 5. It should be noted that spanwise bending modes are divided into flapwise bending modes (whose displacements are predominantly in the direction normal to the middle surface) and edgewise bending modes (whose displacements are predominantly in the direction tangent to the surface) [15] in this paper. The modes $1 \mathrm{~B}$ and $2 \mathrm{~B}$ represent the first and second flapwise bending modes, respectively, and $1 \mathrm{~EB}$ represents the first edgewise bending mode. The modes $1 \mathrm{~T}$ and $2 \mathrm{~T}$ represent the first and second torsional modes, respectively. The chordwise bending modes ( $\mathrm{CB}$, having two or more nodal lines approximately parallel to the pretwisted blade axis) are also found in the successive research. The curves such as $1 \mathrm{~B}, 1 \mathrm{~T}$, and $2 \mathrm{~B}$ and so on in Figures 6-8 are all obtained by using ANSYS Workbench. The curves such as 1st, 2nd, and 3rd are all obtained through numerical calculation.

The first six mode shapes of the FG sandwich blade with $\bar{l}=3, \bar{h}=20, n=2, \bar{h}_{l}=1 / 2$, and $\theta=30^{\circ}$ are given in Figure 9. It can be seen that the results obtained by the two methods are in good agreement. The results in Table 5 show that that the 1 st modes are both of $1 \mathrm{~B}$ mode shape when $\theta=0^{\circ}, 15^{\circ}$, and $30^{\circ}$, but the 2 nd mode is of $1 \mathrm{~T}$ mode shape when $\theta=0^{\circ}$ and $2 \mathrm{~B}$ mode shape when $\theta=15^{\circ}$ and $30^{\circ}$. The same happens with the 1EB mode shape. The reason for this phenomenon is that the stiffness of the FG sandwich blade is changed due to the initial twist in (15).

4.3. Free Vibrations of the FG Sandwich Blade. The effect of the twist angle on the vibration characteristics for the pretwist FG sandwich blade with geometric parameters as $\bar{l}=3$, $\bar{h}=20, n=2$, and $\bar{h}_{l}=1 / 2$ is studied. Table 6 gives the first six nondimensional frequency parameters $\bar{\omega}$ of the FG sandwich blade with varying twist angles $\left(\theta\right.$ from $0^{\circ}$ to $\left.60^{\circ}\right)$, and the relationship between the nondimensional frequency parameters and twist angles for the FG sandwich blade is 
TABLE 7: Nondimensional frequency parameters for the first six modes of the FG sandwich blade with varying thickness ratio $(\bar{l}=3, n=2$, $\bar{h}_{l}=1 / 2$, and $\theta=30^{\circ}$ ).

\begin{tabular}{lccccccccccc}
\hline Mode & 10 & 20 & 30 & 40 & 50 & 60 & 70 & 80 & 90 & 100 \\
\hline 1st & 4.4578 & 4.4743 & 4.4745 & 4.4759 & 4.4903 & 4.5064 & 4.5022 & 4.5115 & 4.5146 & 4.5213 \\
2nd & 24.2636 & 25.4167 & 25.5518 & 25.6696 & 25.7090 & 25.7337 & 25.7686 & 25.8024 & 25.8433 & 25.8342 \\
3rd & 28.2468 & 33.0633 & 38.1941 & 44.8357 & 51.5288 & 59.2127 & 67.3645 & 74.2099 & 77.6186 & 77.2360 \\
4th & 43.9320 & 73.0937 & 75.9033 & 76.1792 & 76.4980 & 76.7083 & 76.7618 & 76.3814 & 82.7484 & 91.1187 \\
5th & 76.6391 & 89.2792 & 113.8445 & 130.9914 & 147.7835 & 151.9612 & 156.271 & 159.8808 & 159.7313 & 159.6019 \\
6th & 87.7677 & 99.5237 & 126.202 & 147.8068 & 155.1631 & 169.1361 & 191.4968 & 207.7979 & 230.7914 & 250.1723 \\
\hline
\end{tabular}

TABLE 8: Nondimensional frequency parameters for the first six modes of the FG sandwich blade with varying aspect ratio $(\bar{h}=20, n=2$, $\bar{h}_{l}=1 / 2$, and $\theta=30^{\circ}$ ).

\begin{tabular}{lccccccccccc}
\hline Mode & 1.00 & 1.25 & 1.50 & 1.75 & 2.00 & 2.25 & 2.50 & 3.00 & 4.00 & 5.00 \\
\hline 1st & 4.4970 & 4.5187 & 4.4946 & 4.4879 & 4.4857 & 4.4847 & 4.4762 & 4.4743 & 4.4643 & 4.4475 \\
2nd & 18.7284 & 20.8402 & 22.6114 & 24.2429 & 25.4037 & 25.4146 & 25.4190 & 25.4167 & 25.4069 & 25.3682 \\
3rd & 25.0639 & 25.2854 & 25.3453 & 25.3845 & 25.9349 & 27.5549 & 29.2911 & 33.0633 & 40.4134 & 48.5665 \\
4th & 35.6083 & 49.9937 & 61.8109 & 68.2541 & 70.4597 & 71.6661 & 72.3847 & 73.0937 & 73.8139 & 73.9446 \\
5th & 45.7901 & 53.9883 & 63.0533 & 69.0347 & 75.8721 & 82.0019 & 87.1364 & 89.2792 & 91.5483 & 93.0731 \\
6th & 58.3492 & 65.8222 & 76.2076 & 82.7443 & 84.4782 & 85.9505 & 88.1750 & 99.5237 & 123.1095 & 147.6281 \\
\hline
\end{tabular}

TABLE 9: Nondimensional frequency parameters for the first six modes of the FG sandwich blade with varying layer thickness ratios $\left(\bar{l}=3, \bar{h}=20, n=2\right.$, and $\left.\theta=30^{\circ}\right)$.

\begin{tabular}{lccccccc}
\hline Mode & $1 / 4$ & $1 / 3$ & $1 / 2$ & $1 / 1$ & $2 / 1$ & $3 / 1$ \\
\hline 1st & 4.2021 & 4.3068 & 4.4743 & 4.7574 & 4.9764 & 5.0854 & 5.1378 \\
2nd & 23.6280 & 24.3536 & 25.4167 & 27.0889 & 28.3771 & 28.9429 & 29.2352 \\
3rd & 30.4576 & 31.3180 & 33.0633 & 35.0677 & 36.7356 & 37.6719 & 38.4396 \\
4th & 68.0698 & 70.1619 & 73.0937 & 78.1350 & 81.7252 & 83.5669 & 84.7660 \\
5th & 83.1451 & 85.6584 & 89.2792 & 95.5154 & 100.6206 & 103.1477 & 104.3400 \\
6th & 92.4498 & 95.1984 & 99.5237 & 106.5238 & 111.4889 & 114.2924 & 116.4530 \\
\hline
\end{tabular}

shown in Figure 6. It can be seen that the resulting curves are very close to the curves obtained by ANSYS in Figure 6(a). The first mode corresponds to the $1 \mathrm{~B}$ vibration mode for all twist angles. The 2nd and 3rd nondimensional frequency parameter curves represent the $1 \mathrm{~T}$ and $2 \mathrm{~B}$ mode shapes when $\theta=0^{\circ}$, and they come close to each other and separate gradually with the twist angle increasing. The two curves are up to the nearest when the twist angle $\theta$ is about $5^{\circ}$; the two modes exchange with each other, and the variation tendencies of nondimensional frequency parameters are also interchanged. The variations for 4th to 6th can be found in Figure 6(b).

It should be noted that the FG sandwich blade designed by the geometric parameters corresponding to the frequency turning points will be prone to internal resonance.

The effect of the thickness ratio on the vibration characteristics for the pretwist FG sandwich blade is studied also. The first six nondimensional frequency parameters $\bar{\omega}$ of the FG sandwich blade with varying thickness ratios ( $\bar{h}$ from 10 to 100 ) is given in Table 7 . Figure 7 shows the relationship between the nondimensional frequency parameters and the thickness ratio. It can be found that the nondimensional frequency parameters of $1 \mathrm{~B}, 2 \mathrm{~B}, 3 \mathrm{~B}$, and $4 \mathrm{~B}$ modes remain essentially unchanged when the thickness ratio varies from 10 to 100 . The nondimensional frequency parameters of $1 \mathrm{~T}$, $2 \mathrm{~T}$, and $1 \mathrm{~EB}$ modes tend to increase with increasing thickness ratio. On the other side, the $3 \mathrm{rd}$ and 4 th frequency parameter loci have an obvious veering at $\bar{h}=85$, and the mode shapes exchanged between $1 \mathrm{~T}$ and $3 \mathrm{~B}$. The same situation can be seen on the 5th and 6th modes, and higher-order frequency parameter locus veering happens more frequently.

The nondimensional frequency parameters $\bar{\omega}$ for the first six modes of the FG sandwich blade with varying aspect ratios ( $\bar{l}$ from 1 to 5 ) are given in Table 8 to study the effect of the aspect ratio on the vibration characteristics for the pretwist FG sandwich blade. Figure 8 shows the relationships between the first six frequency parameters, the corresponding mode shapes, and the aspect ratio. It is obvious that the 1 st mode corresponds to the $1 \mathrm{~B}$ vibration mode for all aspect ratios, and the value of the frequency parameter varies little. The 2 nd and 3rd modes exchange with each other, and the 


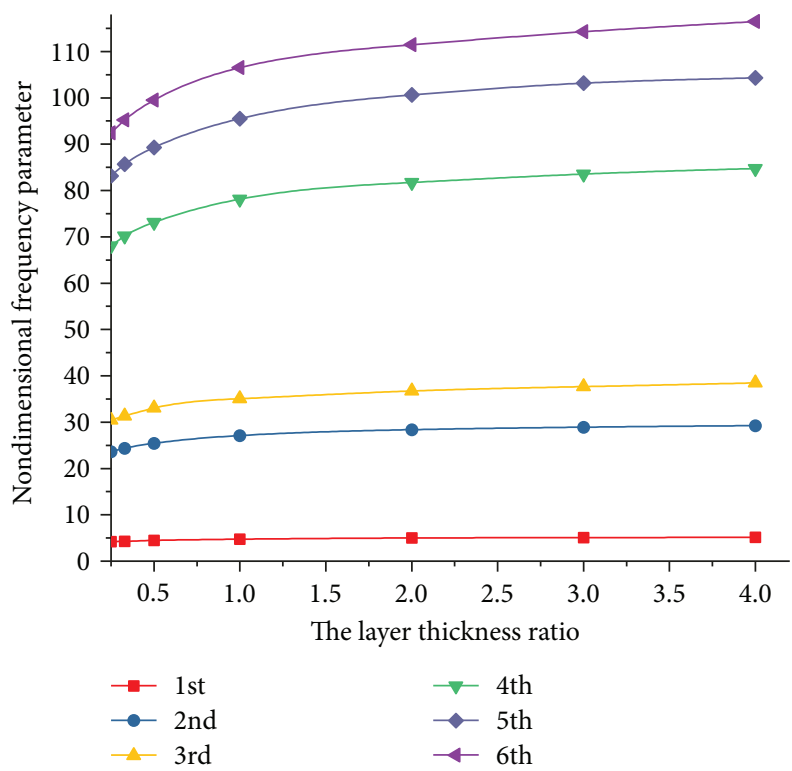

FIGURE 10: Variations of nondimensional frequency parameters with the layer thickness ratio $\left(\bar{l}=3, \bar{h}=20, n=2\right.$, and $\left.\theta=30^{\circ}\right)$.

TABLE 10: Nondimensional frequency parameters for the first six modes of the FG sandwich blade with varying scalar parameters of volume fraction $\left(\bar{l}=3, \bar{h}=20, \bar{h}_{l}=1 / 2\right.$, and $\left.\theta=30^{\circ}\right)$.

\begin{tabular}{lcccccccccccc}
\hline Mode & 0.0 & 0.1 & 0.2 & 0.3 & 0.4 & 0.5 & 1.0 & 2.0 & 3.0 & 4.0 & 5.0 \\
\hline 1st & 3.3606 & 3.5795 & 3.6493 & 3.8619 & 3.8665 & 3.9753 & 4.2248 & 4.4743 & 4.5659 & 4.6931 & 4.7617 \\
2nd & 19.1226 & 20.0849 & 20.7619 & 21.4358 & 21.9111 & 22.3903 & 23.8825 & 25.4167 & 26.1362 & 26.5972 & 26.8850 \\
3rd & 24.9882 & 26.0961 & 26.9426 & 27.6920 & 28.2425 & 28.7613 & 30.7357 & 33.0633 & 33.7313 & 34.2049 & 34.8816 \\
4th & 55.8597 & 58.2781 & 60.3344 & 62.0908 & 63.5612 & 64.7114 & 68.9569 & 73.0937 & 75.1927 & 76.5550 & 77.6652 \\
5th & 71.5372 & 73.7012 & 75.7393 & 77.3010 & 78.8343 & 80.0439 & 84.5755 & 89.2792 & 91.5699 & 93.1665 & 93.7638 \\
6th & 75.5421 & 79.2376 & 81.6705 & 84.0076 & 85.8903 & 86.6503 & 93.4294 & 99.5237 & 102.7955 & 104.0268 & 106.0757 \\
\hline
\end{tabular}

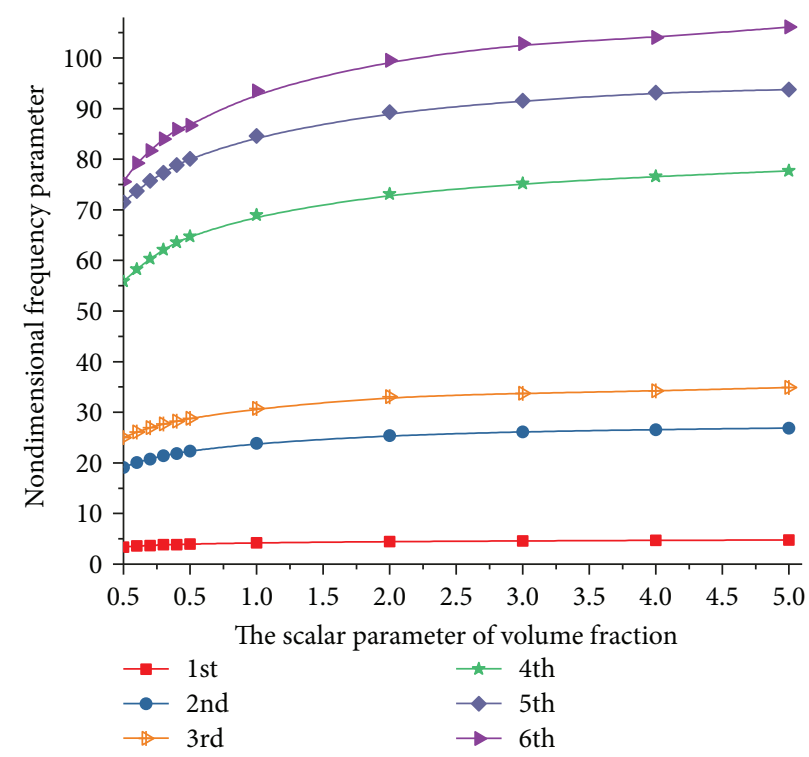

FIGURE 11: Variations of nondimensional frequency parameters with the scalar parameter of volume fraction $\left(\bar{l}=3, \bar{h}=20, \bar{h}_{l}=1 / 2\right.$, and $\left.\theta=30^{\circ}\right)$. 
TABLE 11: Nondimensional frequency parameters for the first six modes of the FG sandwich blade with varying nondimensional rotation velocities $\left(\bar{l}=3, \bar{h}=20, n=2, \bar{h}_{l}=1 / 2, \theta=30^{\circ}, \bar{r}=1\right.$, and $\left.\varphi=45^{\circ}\right)$.

\begin{tabular}{lccccccccccccc}
\hline Mode & 0 & 1 & 2 & 3 & 4 & 5 & 6 & 7 & 8 & 9 & 10 & 11 \\
\hline 1st & 4.4743 & 6.9030 & 11.2340 & 15.8419 & 20.5025 & 25.1652 & 29.8098 & 34.4146 & 38.9622 & 43.4424 & 47.8358 & 52.1334 \\
2nd & 25.4167 & 28.5235 & 34.0383 & 35.1206 & 36.4308 & 38.1374 & 40.2129 & 42.4223 & 44.6896 & 47.3581 & 50.0889 & 52.8240 \\
3rd & 33.0633 & 33.0979 & 35.8589 & 45.2978 & 55.2322 & 64.5633 & 72.3156 & 78.0880 & 82.3864 & 85.9246 & 89.1536 & 92.3135 \\
4th & 73.0937 & 76.2806 & 80.9215 & 84.9748 & 88.5518 & 92.9797 & 99.3391 & 108.0164 & 118.4814 & 130.0075 & 142.1141 & 154.5548 \\
5th & 89.2792 & 92.7844 & 96.5525 & 106.6897 & 114.5673 & 121.7465 & 129.8789 & 138.6539 & 147.8984 & 157.7082 & 167.8623 & 178.1525 \\
6th & 99.5237 & 100.9778 & 103.9269 & 108.5284 & 120.9391 & 137.5384 & 155.5090 & 174.2894 & 193.5633 & 213.0626 & 232.5021 & 250.6452 \\
\hline
\end{tabular}

vibration tendencies of their frequency loci also exchange near $\bar{l}=2$. The change of the 4 th to 6 th nondimensional frequency parameters is relatively complicated. The mode shapes include $3 \mathrm{~B}, 2 \mathrm{~T}, 1 \mathrm{~EB}$, and $1 \mathrm{CB}$. For example, the 4 th to 6 th mode shapes are $1 \mathrm{CB}, 2 \mathrm{~T}$, and $1 \mathrm{~EB}$ when $\bar{l}=1 ; 2 \mathrm{~T}$, $1 \mathrm{~EB}$, and $1 \mathrm{CB}$ when $\bar{l}=1.5$; and $1 \mathrm{~EB}, 2 \mathrm{~T}$, and $3 \mathrm{~B}$ when $\bar{l}=1.75$. All in all, the mode shape corresponding to the same order frequency is very sensitive to the variation of small aspect ratios, especially when $\bar{l}<2$. The tendencies of their frequency parameters become relatively stable until $\bar{l}>2.5$.

Table 9 and Figure 10 show the variations of the first six nondimensional frequency parameters of the FG sandwich blade with the layer thickness ratio. Table 10 and Figure 11 show the relationships between the first six nondimensional frequency parameters and the scalar parameter of the volume fraction. It can be found that the content of ceramic increases with increasing layer thickness ratio and scalar parameter of the volume fraction, and the frequency parameters of all modes increase, respectively.

Table 11 shows the first six nondimensional frequency parameters of the FG sandwich blade for the nondimensional rotation velocity with the range of $\bar{\Omega} 0$ to 11 (about $15,000 \mathrm{rpm}$ ). A typical Campbell diagram is plotted to study the variation trend of the nondimensional frequency parameters for varying rotation velocities in Figure 12. Frequency locus veering is observed as expected: the 1 st and 2 nd frequency modes exchange between $1 \mathrm{~B}$ and $1 \mathrm{~T}$ around the rotation velocity $\bar{\Omega}=11$; the 2 nd and 3 rd frequency modes exchange between $2 \mathrm{~B}$ and $1 \mathrm{~T}$ around $\bar{\Omega}=2$; the 5 th and 6 th frequency modes exchange between $1 \mathrm{~EB}$ and $2 \mathrm{~T}$ around $\bar{\Omega}=3$; and the 7 th and 8 th frequency modes exchange between $4 \mathrm{~B}$ and $3 \mathrm{~T}$ around $\bar{\Omega}=1$ and exchange between $3 \mathrm{~T}$ and $1 \mathrm{CB}$ again around the rotation velocity $\bar{\Omega}=9$. In addition, the 8th frequency locus can be seen veering at $\bar{\Omega}=4$ and the mode shape is changed from $4 \mathrm{~B}$ to $1 \mathrm{~EB}$. It can be seen clearly that higher-order frequencies tend to have multiple frequency veering with the mode shapes exchanging and coupling.

Figure 13 shows the effects of the stagger angle for the first six nondimensional frequency parameters with varying rotation velocities. It can be found that the stagger angle has a predominant influence in the 1st and 2nd modes, and the effect is very marginal in higher modes. The effect of the stagger angle has little influence on all frequencies when

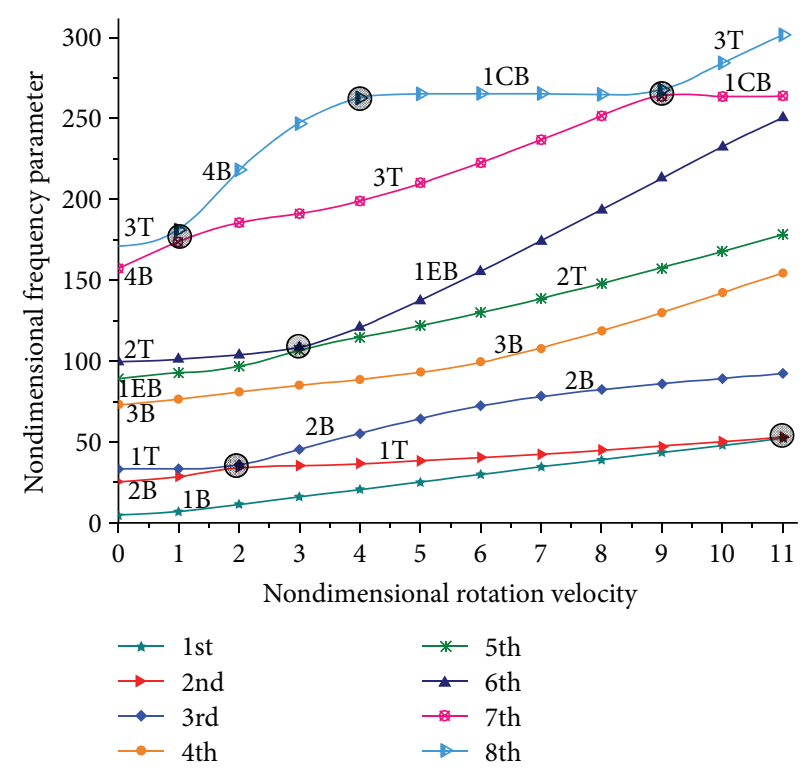

FIgURE 12: A Campbell diagram of the FG sandwich blade $(\bar{l}=3$, $\bar{h}=20, n=2, \bar{h}_{l}=1 / 2, \theta=30^{\circ}, \bar{r}=1$, and $\varphi=45^{\circ}$ ).

the rotation velocity is low. The reason is that the centrifugal force has little effect on the stiffening and softening when the rotating speed is low, and the influence becomes more and more obvious with the rotating speed increasing.

\section{Conclusion}

In this paper, a dynamic model based on the thick shell theory is developed to investigate the free vibration behavior of functionally graded sandwich pretwisted blades. The validation of the homogeneous material and FG sandwich blade is performed by comparison to the literature and ANSYS results, both showing good agreement.

Interesting frequency veering and the associated mode shift phenomena are found and discussed for different twist angles, thickness ratios, and aspect ratios in static natural frequency analysis. The results show that the fundamental mode is flapwise bending in every case. The mode shapes of the 2nd to 6th orders are changing when different geometric parameters are used; especially, the thickness ratio is $15-50$ 

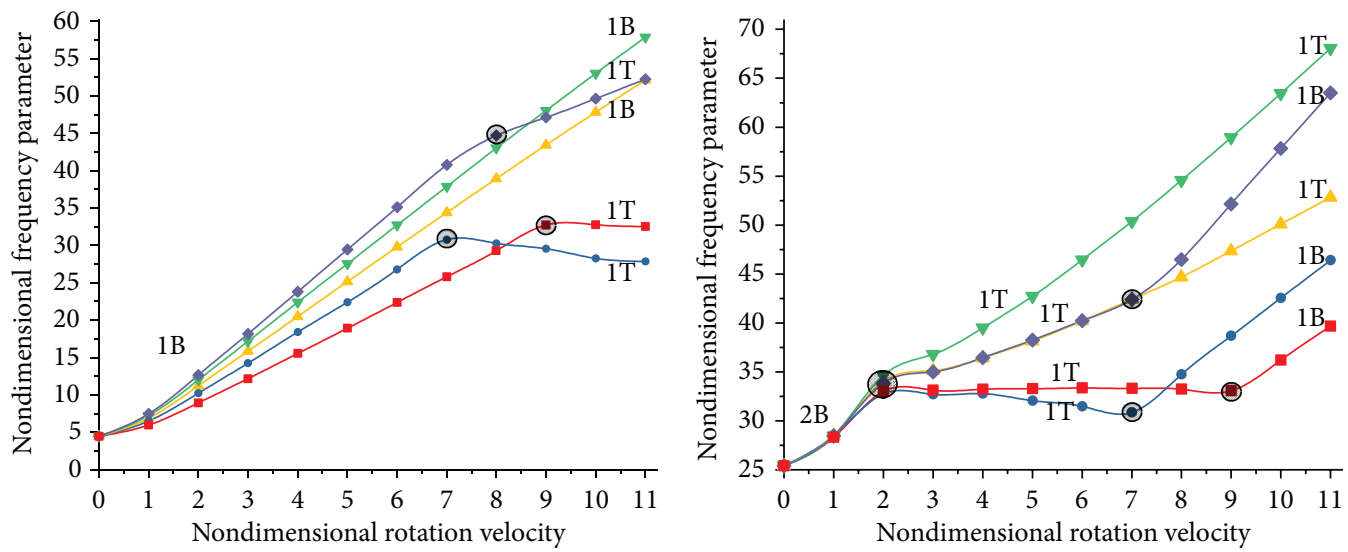

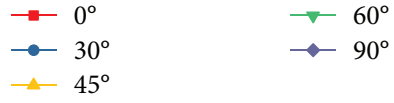

(a)

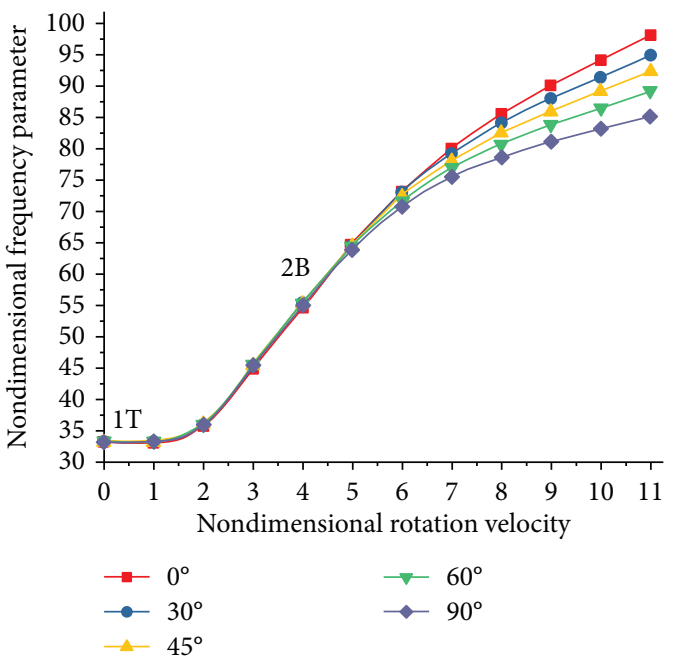

(c)
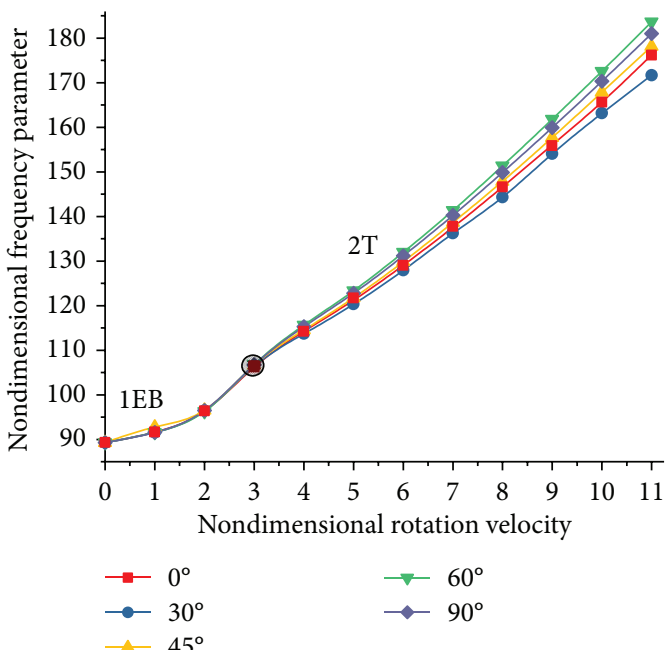

(e)

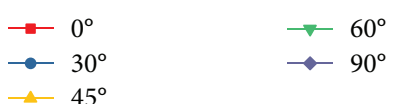

(b)

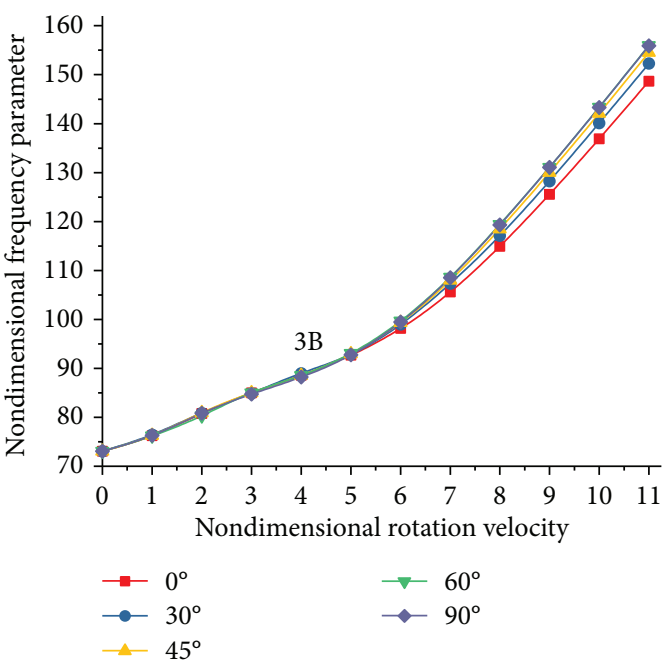

(d)
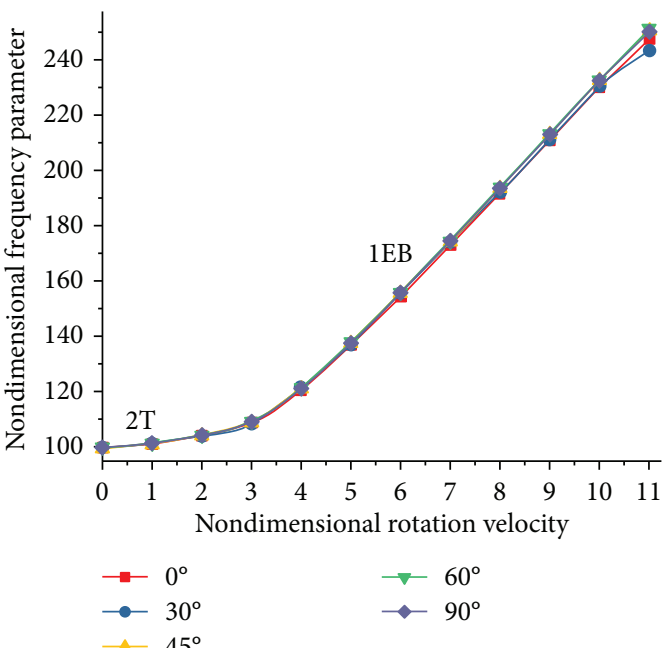

(f)

FIGURE 13: Variations of the first six frequency parameters with the rotation velocity and stagger angles $\left(\bar{l}=3, \bar{h}=20, n=2, \bar{h}_{l}=1 / 2, \theta=30^{\circ}\right.$, and $\bar{r}=1$ ). (a) 1st mode, (b) 2nd mode, (c) 3rd mode, (d) 4th mode, (e) 5th mode, and (f) 6th mode. 
and the aspect ratio is 1.0-2.5. Higher-order frequency loci $(\geq 4)$ may veer more than once between the bending and torsional vibrations of the FG sandwich blade.

The stagger angle is considered to study the dynamic frequency with varying rotation velocities. The results show that the frequency modes have more complicated shapes and stronger coupling in the rotating FG sandwich blade system. The Campbell diagram reveals that the rotating velocity, especially high rotating velocity, has a significant impact on the variations of frequency loci and mode shapes.

\section{Conflicts of Interest}

The authors declare that they have no conflicts of interest.

\section{Acknowledgments}

The authors acknowledge the financial support of the National Natural Science Foundation of China through Grant no. 11472056, Fundamental Research Program of Shenzhen Municipality no. JCYJ20160608153749600, and Scientific Research Project of Beijing Municipal Commission of Education nos. KM201811232002 and KM201711232002.

\section{References}

[1] A. Leissa and K. I. Jacob, "Three-dimensional vibrations of twisted cantilevered parallelepipeds," Journal of Applied Mechanics, vol. 53, no. 3, pp. 614-618, 1986.

[2] H. H. Yoo, J. H. Park, and J. Park, "Vibration analysis of rotating pre-twisted blades," Computers and Structures, vol. 79, no. 19, pp. 1811-1819, 2001.

[3] N. K. Chandiramani, C. D. Shete, and L. I. Librescu, "Vibration of higher-order-shearable pretwisted rotating composite blades," International Journal of Mechanical Sciences, vol. 45, no. 12, pp. 2017-2041, 2003.

[4] E. Carrera, M. Filippi, and E. Zappino, "Free vibration analysis of rotating composite blades via Carrera Unified Formulation," Composite Structures, vol. 106, no. 106, pp. 317-325, 2013.

[5] M. S. Qatu and A. W. Leissa, "Vibration studies for laminated composite twisted cantilever plates," International Journal of Mechanical Sciences, vol. 33, no. 11, pp. 927-940, 1991.

[6] S. Mohamed Nabi and N. Ganesan, "Comparison of beam and plate theories for free vibrations of metal matrix composite pre-twisted blades," Journal of Sound and Vibration, vol. 189, no. 2, pp. 149-160, 1996.

[7] H. H. Yoo and J. Chung, "Dynamics of rectangular plates undergoing prescribed overall motion," Journal of Sound and Vibration, vol. 239, no. 1, pp. 123-137, 2001.

[8] X. X. Hu, T. Sakiyama, and K. Itakura, "A new numerical procedure for free vibrations of pretwisted plates," Archive of Applied Mechanics, vol. 72, no. 4-5, pp. 330-341, 2002.

[9] S. H. Hashemi, S. Farhadi, and S. Carra, "Free vibration analysis of rotating thick plates," Journal of Sound and Vibration, vol. 323, no. 1-2, pp. 366-384, 2009.

[10] S. K. Sinha and K. E. Turner, "Natural frequencies of a pretwisted blade in a centrifugal force field," Journal of Sound and Vibration, vol. 330, no. 11, pp. 2655-2681, 2011.

[11] J. Sun, L. Kari, and I. Lopez Arteaga, "A dynamic rotating blade model at an arbitrary stagger angle based on classical plate theory and the Hamilton's principle," Journal of Sound and Vibration, vol. 332, no. 5, pp. 1355-1371, 2013.

[12] J. S. Rao and K. Gupta, "Free vibrations of rotating small aspect ratio pretwisted blades," Mechanism and Machine Theory, vol. 22, no. 2, pp. 159-167, 1987.

[13] J. Sun, I. Lopez Arteaga, and L. Kari, "General shell model for a rotating pretwisted blade," Journal of Sound and Vibration, vol. 332, no. 22, pp. 5804-5820, 2013.

[14] S. K. Sinha and R. P. Zylka, "Vibration analysis of composite airfoil blade using orthotropic thin shell bending theory," International Journal of Mechanical Sciences, vol. 121, no. 2, pp. 90-105, 2017.

[15] R. E. Kielb, A. W. Leissa, J. C. Macbain, and K. S. Carney, "Joint research effort on vibrations of twisted plates, phase 1: final results," Tech. Rep. 1150, NASA Lewis Research Center, Cleveland, OH, USA, 1985.

[16] R. W. Claassen and C. J. Thorne, "Vibrations of a rectangular cantilever plate," Journal of the Aerospace Sciences, vol. 29, no. 11, pp. 1300-1305, 1962.

[17] D. Afolabi and O. Mehmed, "On curve veering and flutter of rotating blades," Journal of Engineering for Gas Turbines and Power, vol. 116, no. 3, pp. 702-708, 1994.

[18] H. H. Yoo and C. Pierre, "Modal characteristic of a rotating rectangular cantilever plate," Journal of Sound and Vibration, vol. 259, no. 1, pp. 81-96, 2003.

[19] H. H. Yoo, S. K. Kim, and D. J. Inman, "Modal analysis of rotating composite cantilever plates," Journal of Sound and Vibration, vol. 258, no. 2, pp. 233-246, 2002.

[20] S. Y. Oh, L. Librescu, and O. Song, "Vibration of turbomachinery rotating blades made-up of functionally graded materials and operating in a high temperature field," Acta Mechanica, vol. 166, no. 1-4, pp. 69-87, 2003.

[21] M. N. V. Ramesh and N. Mohan Rao, "Free vibration analysis of pre-twisted rotating FGM beams," International Journal of Mechanics and Materials in Design, vol. 9, no. 4, pp. 367383, 2013.

[22] Y. Oh and H. H. Yoo, "Vibration analysis of rotating pretwisted tapered blades made of functionally graded materials," International Journal of Mechanical Sciences, vol. 119, pp. 68-79, 2016.

[23] J. L. Mantari and E. V. Granados, "Dynamic analysis of functionally graded plates using a novel FSDT," Composites Part B: Engineering, vol. 75, pp. 148-155, 2015.

[24] L. Li and D. G. Zhang, "Free vibration analysis of rotating functionally graded rectangular plates," Composite Structures, vol. 136, pp. 493-504, 2016.

[25] J. Sun, I. Lopez Arteaga, and L. Kari, "Dynamic modeling of a multilayer rotating blade via quadratic layerwise theory," Composite Structures, vol. 99, pp. 276-287, 2013.

[26] D. Cao, B. Liu, M. Yao, and W. Zhang, "Free vibration analysis of a pre-twisted sandwich blade with thermal barrier coatings layers," Science China Technological Sciences, vol. 60, no. 11, pp. 1747-1761, 2017.

[27] A. M. A. Neves, A. J. M. Ferreira, E. Carrera et al., "Static, free vibration and buckling analysis of isotropic and sandwich functionally graded plates using a quasi-3d higher-order shear deformation theory and a meshless technique," Composites Part B: Engineering, vol. 44, no. 1, pp. 657-674, 2013.

[28] T. Tsuji and T. Sueoka, "Free vibrations of pre-twisted plates: fundamental theory: vibration, control engineering, 
engineering for industry," JSME International Journal, vol. 30, no. 264, pp. 958-962, 1987.

[29] M. S. Qatu, R. W. Sullivan, and W. Wang, "Recent research advances on the dynamic analysis of composite shells: 20002009," Composite Structures, vol. 93, no. 1, pp. 14-31, 2010.

[30] M. Amabili, Nonlinear Vibrations and Stability of Shells and Plates, Cambridge University Press, 2008.

[31] M. S. Qatu, Vibration of Laminated Shells and Plates, Elsevier, San Diego, CA, USA, 2004.

[32] J. N. Reddy, Mechanics of Laminated Composite Plates and Shells: Theory and Analysis, CRC Press, 2004. 


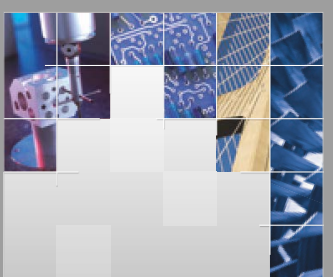

\section{Enfincering}
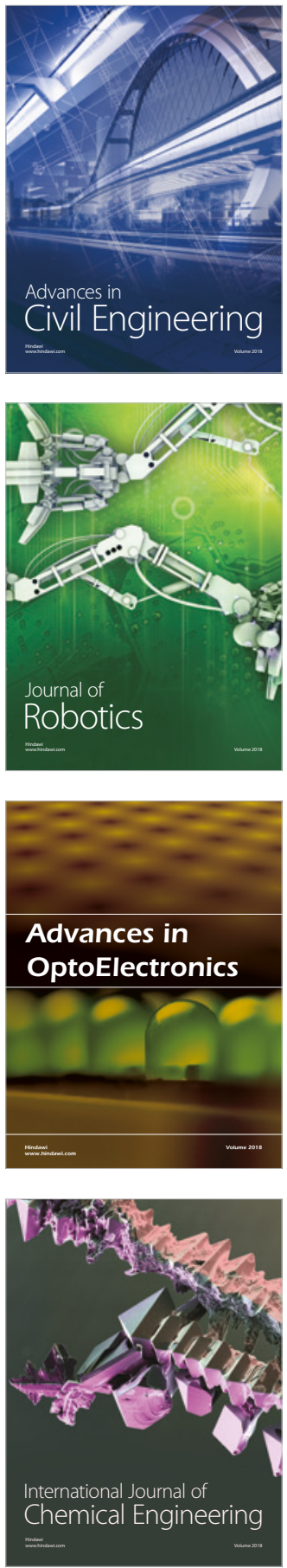

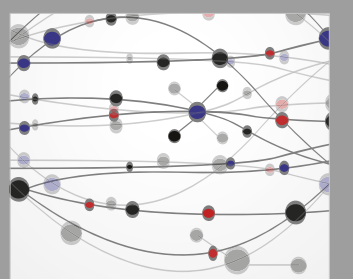

\section{Rotating \\ Machinery}

The Scientific World Journal

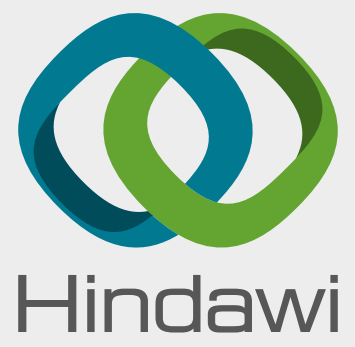

Submit your manuscripts at

www.hindawi.com
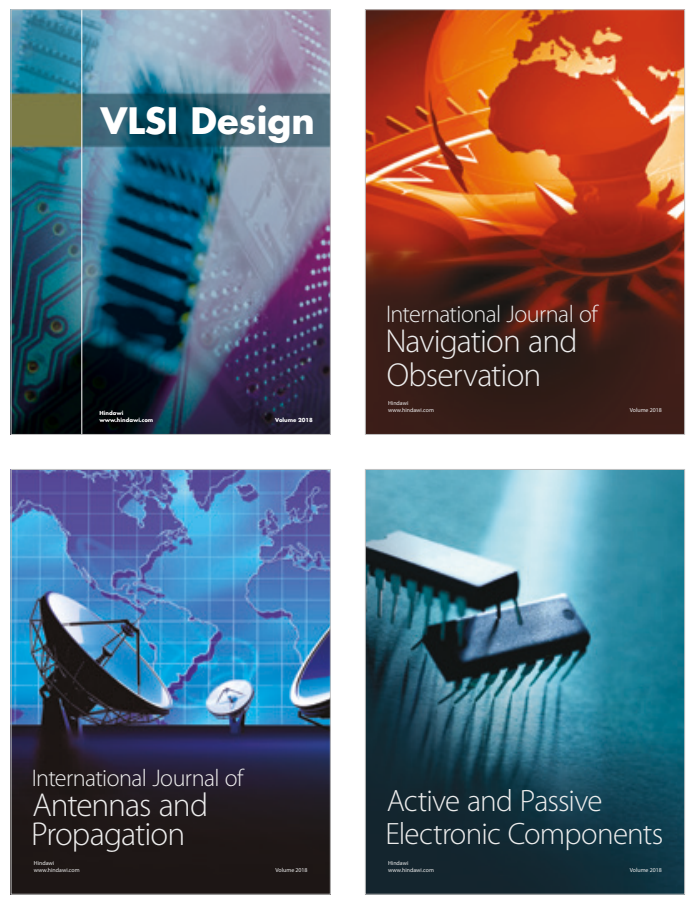
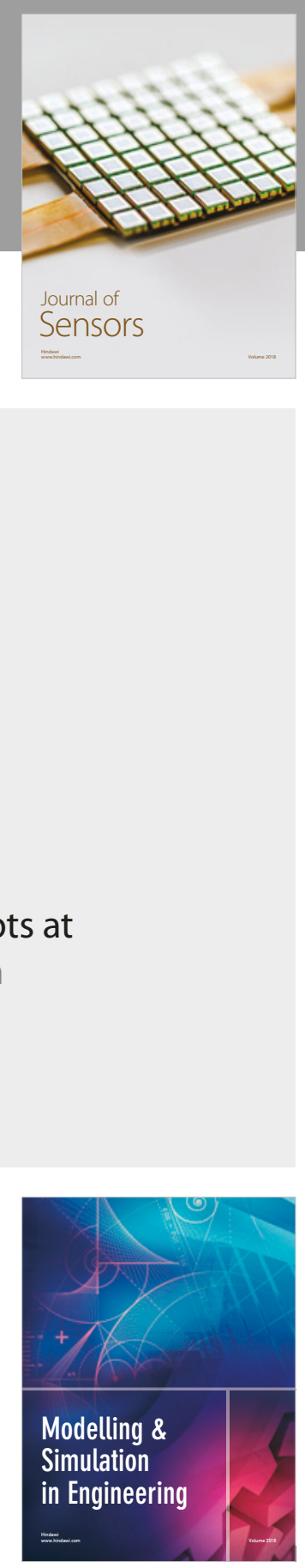

\section{Advances \\ Multimedia}
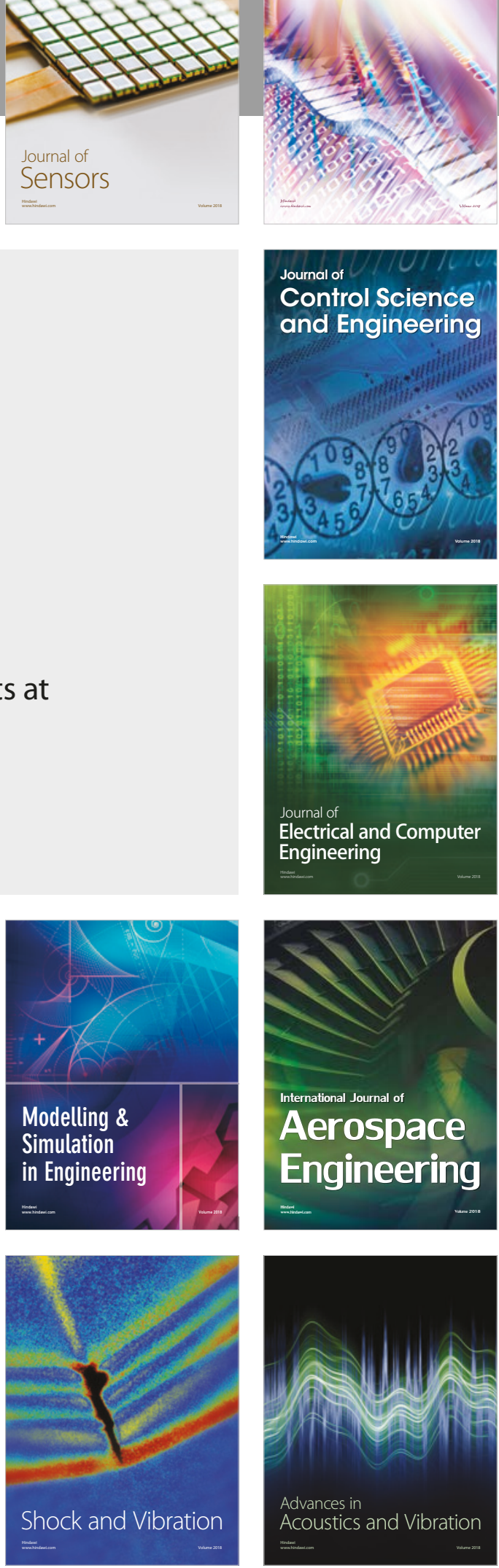